\title{
Modellierung pluvialer Sturzfluten - Anforderungen und Sensitivitäten der 2D-hydraulischen Modellierung
}

\author{
Andreas Huber · Simon Lumassegger · Bernhard Kohl · Yvonne Spira · Felix Weingraber · Stefan Achleitner $(D)$
}

Angenommen: 22. Februar 2021 / Online publiziert: 15. März 2021

(C) Der/die Autor(en) 2021

\begin{abstract}
Zusammenfassung Lokale Starkregen und der daraus resultierende Oberflächenwasserabfluss führten in den letzten Jahren vermehrt $\mathrm{zu}$ pluvialen Sturzfluten (Hangwässern) verbunden mit Überflutungen und Schäden. Auch in Österreich kam es vermehrt zu derartigen Ereignissen bei denen nicht nur urban geprägte Gebiete, sondern auch vielfach ländliche Gebiete mit überwiegend landwirtschaftlicher Nutzung betroffen waren. Überflutungsmodellierungen sowie daraus abgeleitete Risikoabschätzungen und Maßnahmenplanungen stellen wichtige Werkzeuge dar, um zukünftig Schäden abzuwenden bzw. zu minimieren. In den Projekten RAINMAN, AQUACLEW und SAFFERCC kamen an mehreren Fallbeispielen unterschiedliche 2D-hydrodynamische, numerische (2D-HN) Oberflächenabflussmodelle zur Anwendung, um Überflutungsflächen, Wasserstände und Fließgeschwindigkeiten abzuschätzen. Neben dem Vergleich unter-
\end{abstract}

DI A. Huber · S. Lumassegger . Assoz.-Prof. DI Dr. S. Achleitner (殴 Arbeitsbereich für Wasserbau, Universität Innsbruck,

Technikerstraße 13, 6020 Innsbruck, Österreich

stefan.achleitner@uibk.ac.at

MR Mag. Dr. B. Kohl

Institut für Naturgefahren BFW - Bundesforschungs- und Ausbildungszentrum für Wald, Naturgefahren und Landschaft, Rennweg 1, Hofburg, 6020 Innsbruck, Österreich

DI Dr. Y. Spira

Team Oberflächengewässer, Umweltbundesamt, Spittelauer Lände 5, 1090 Wien, Österreich

Mag. F. Weingraber

Abt. Wasserwirtschaft, Gruppe

Hochwasserschutz, Amt der Oberösterreichischen

Landesregierung, Kärntner Straße 10-12, 4021 Linz, Österreich schiedlicher Modelle wurde die Sensitivität von Modellparametern bei unterschiedlichen Bemessungsszenarien erfasst. Hinsichtlich der Abflussbildung wurde die Auswirkung geänderter $\mathrm{Ab}$ flussbeiwerte (Landnutzungsänderung, Unsicherheiten etc.) auf die Überflutungsbilder erhoben. Analog wurden Modellsensitivitäten bei den Prozessen der Abflusskonzentration betrachtet. Der Einfluss von Modellauflösung, Parametrisierung der Rauheit, Implementierung hydraulisch relevanter Strukturen (Gebäude, Mauern und Durchlässe) wurden mit unterschiedlichen hydraulischen Modellen getestet. Zur Anwendung kamen dabei rasterbasierte Modelle und Ansätze mit freien/ unstrukturierten Netzen.

Schlüsselwörter 2D-gekoppelte Modellierung · Pluviale Sturzfluten . Modellparametrisierung

Sensitivitätsanalyse

Modeling of pluvial flash floods requirements and sensitivities of 2D hydraulic modeling

Abstract In recent years, local heavy rainfall and the resulting surface water runoff have increasingly led to pluvial flash floods and associated damages. In Austria, too, such events occurred more frequently, affecting not only urban areas but also rural areas with predominantly agricultural land use. Flood modeling and the resulting risk assessment and planning of measures are important tools to prevent or minimize damages in the future. In the RAINMAN, AQUACLEW and SAFFER-CC projects, different 2Dhydrodynamic numerical (2D-HN) surface runoff models were applied to several case studies to estimate inundation areas, water levels and flow velocities. In addition to comparing different models, the sensitivity of model parameters under different design scenarios was investigated. Regarding runoff generation, the effect of changed runoff coefficients (land use change, uncertainties, ...) on the flooding patterns was analyzed. Similarly, model sensitivities were considered in the processes of runoff concentration. The influence of model resolution, roughness parameterization and implementation of hydraulically relevant structures (buildings, walls and culverts) were tested with different hydraulic models. Raster-based and free/ unstructured mesh approaches were applied.

Keywords 2D coupled modeling . Pluvial flash flooding - Model parameterization $\cdot$ Sensitivity analysis

\section{Einleitung}

Überflutungen durch oberflächlich abfließendes Wasser infolge von Starkregenereignissen sind in den letzten Jahren in Österreich und im benachbarten Ausland vermehrt in den Fokus des öffentlichen, medialen und wissenschaftlichen Interesses gerückt (u. a. Achleitner et al. 2020a; LUBW 2016; Bernet et al. 2017; Lumassegger et al. 2016a, b; Zahnt et al. 2018; Kipfer et al. 2018; Starl 2020). In den letzten Jahren konnten wiederholt Schadensereignisse infolge kleinräumiger Starkregen beobachtet werden. Im Gegensatz zu Hochwässern an Flüssen (fluviale Hochwässer) sind Überflutungen durch Oberflächenabfluss nicht auf relativ gut abgrenzbare Gebiete entlang von bestehenden Wasserläufen beschränkt. Diese sogenannten pluvialen Überflutungen treten häufig mit kurzer Vorwarnzeit und in vielen Fällen weit abseits von Gewässern auf (Strehz et al. 2015) und stellen ein schwer kalkulierbares Überflutungsrisiko dar. Potenziell sind alle Regionen Österreichs gefährdet, wobei der exakte Ort und Zeitpunkt des Auftretens nur schwer vorhersagbar sind. Aktuelle Untersuchungen aus der Klimaforschung gehen zudem davon aus, dass die Niederschlagsintensitäten von Starkregen mit kurzer Dauer in Zukunft 


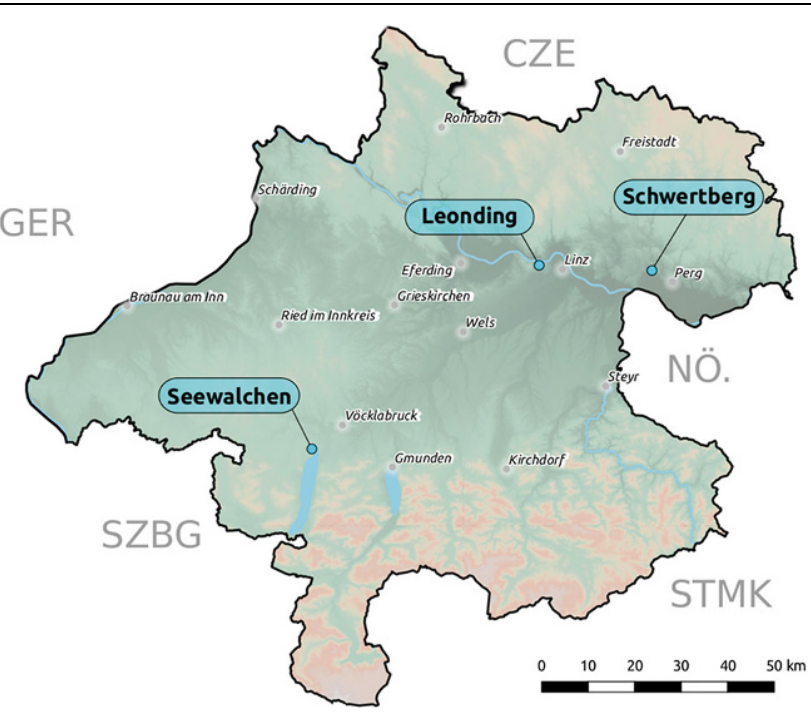

Abb. 1 Übersicht über die Pilotgebiete in Oberösterreich

noch weiter zunehmen könnten (u.a. Formayer und Kromp-Kolb 2009; Berg et al. 2013; Nissen und Ulbrich 2017; Giorgi et al. 2019; Becker 2019). Informationen über eine mögliche Gefährdung durch Oberflächenabfluss sind dementsprechend als Planungsgrundlage für zukünftige Bauvorhaben und die Realisierung von Schutz- und Vorsorgemaßnahmen für Bestandsbauten von großem Interesse (Humer et al. 2015; Achleitner et al. 2020a).

In den letzten Jahren wurden verschiedene Methoden vorgeschlagen und angewendet, um die Überflutungsgefährdung durch Oberflächenabfluss nach Starkregenfällen zu beurteilen. Eine Ersteinschätzung der Gefährdungssituation kann oft auf Basis lokaler Expertise und der Analyse vergangener Ereignisse erfolgen. Bekannte und offensichtliche Problembereiche können mit dieser Methode identifiziert werden, die Aussagekraft hinsichtlich möglicher zukünftiger oder bisher unbeobachteter Gefährdungsbereiche ist aber begrenzt. Eine detaillierte Analyse der Gefährdung durch pluviale Überflutungen erfordert eine zweidimensionale hydrodynamische, numerische (2DHN) Gefährdungsanalyse.

Die Gefährdungsanalyse mit 2DHN-Modellen ermöglicht im Gegensatz zu rein topografiebasierten Methoden (z. B. GIS basierte „Fließwegkarten“ basierend auf unterschiedlichen flow accumulation-Algorithmen; O'Callaghan und Mark 1984; Quinn et al. 1991; Holmgren 1994; Tarboton 1997) die Darstellung von Wasserspiegellagen und Überflutungstiefen. Mit 2D-HN-
Modellen lassen sich zudem die möglichen auftretenden Fließgeschwindigkeiten darstellen und unterschiedliche Szenarien differenziert analysieren. Der Einsatz von 2D-HN-Modellen ist aufgrund dieser Vorteile mittlerweile Stand der Technik.

Während 2D-HN-Modelle für Anwendungen im Bereich fluvialer Prozesse schon seit langem etabliert sind, ist der Einsatz dieser Modelle für die Modellierung pluvialer Sturzfluten noch ein relativ neues Anwendungsfeld. Mittlerweile gibt es eine wachsende Anzahl von Softwarepaketen mit unterschiedlich implementierten hydrodynamischen Grundgleichungen, Rechennetzgeometrien, Lizenzierungen und Funktionalitäten.

In diesem Beitrag werden Untersuchungen mit verschiedenen 2D-HNModellen beschrieben, die im Rahmen der Projekte RAINMAN (Interreg Central Europe), AQUACLEW (EU-ER4 CS) und SAFFER-CC (ACRP-Klima und Energiefonds) zur Gefährdungsanalyse für pluviale Sturzfluten in oberösterreichischen Pilotgemeinden durchgeführt wurden. Bezüglich der Abflussbildung wurde der Einfluss von veränderten Abflussbeiwerten (Landnutzungsänderung, Unsicherheit etc.) auf Hochwasserbilder untersucht. Auch Modellsensitivitäten bei Abflusskonzentrationsprozessen wie dem Einfluss der Modellauflösung, der Rauigkeitsparametrisierung oder der Implementierung von hydraulisch relevanten Strukturen (Gebäude, Mauern und Durchlässe) wurde mit den unterschiedlichen hydraulischen Modellen untersucht.

\section{Pilotgebiete}

Die Untersuchungen wurden in den drei Pilotgemeinden Schwertberg, Seewalchen und Leonding in Oberösterreich durchgeführt, in denen es in den vergangenen Jahren zu pluvialen Überflutungen (Hangwasserabflüssen) infolge von Starkregenereignissen gekommen ist. Die durchgeführten Untersuchungen umfassen ausgewählte Einzugsgebiete in den genannten Gemeinden. In den Pilotgebieten Seewalchen und Schwertberg umfassten die Sensitivitätsanalysen (a) Abflussbildung, (b) Auflösung der Netzgeometrie und des Höhenmodells (DGM) und (c) Rauheit. Im Pilotgebiet Schwertberg wurden zusätzlich (d) hydraulisch relevante Strukturen untersucht. In Leonding wurden ergänzende Beregnungsversuche durchgeführt (Abb. 1).

Die Größe der modellierten Gebiete beträgt in Seewalchen ca. $2,6 \mathrm{~km}^{2}$ und in Schwertberg ca. $2 \mathrm{~km}^{2}$. Als Datenbasis für die Erstellung der 2D-HN-Modelle wurden folgende Datenquellen verwendet:

- DGM mit $0.5 \mathrm{~m}$ Rasterauflösung aus Laserscanbefliegungen (Land Oberösterreich),

- Nutzflächen aus der digitalen Katastralmappe (DKM) (Bundesamt für Eich- und Vermessungswesen, BEV),

- Orthofotos Zeitraum 2015-2017 (Open Data, Land Oberösterreich).

Für die Modellerstellung wurden Gebäudeflächen aus der DKM übernommen, sofern ein visueller Vergleich mit den Orthofotos eine Übereinstimmung mit dem Realbestand ergab. Gebäude, die im Datenbestand der DKM nicht vorhanden waren oder deren Geometrie den Realbestand nach Orthofoto nicht wiederspiegelt, wurden händisch auf Basis der sichtbaren Dachflächen aus dem Orthofoto digitalisiert. Die Nutzflächen aus der DKM wurden auch als Grundlage für die Abgrenzung verschiedener Landnutzungs- bzw. Landbedeckungseinheiten für die Modellierung herangezogen. Die Nutzflächen aus der DKM wurden dabei aggregiert und, wo nötig, nachbearbeitet und für die Modellierung vereinfacht in $7 \mathrm{Ka}$ tegorien zusammengefasst. Die aggregierte und überarbeitete Landnutzung für die beiden Untersuchungsgebiete in Schwertberg und Seewalchen ist in Abb. 2 dargestellt. Landwirtschaftliche Flächen mit den übergeordneten Nutzungsarten Acker, Wiese oder Wei- 


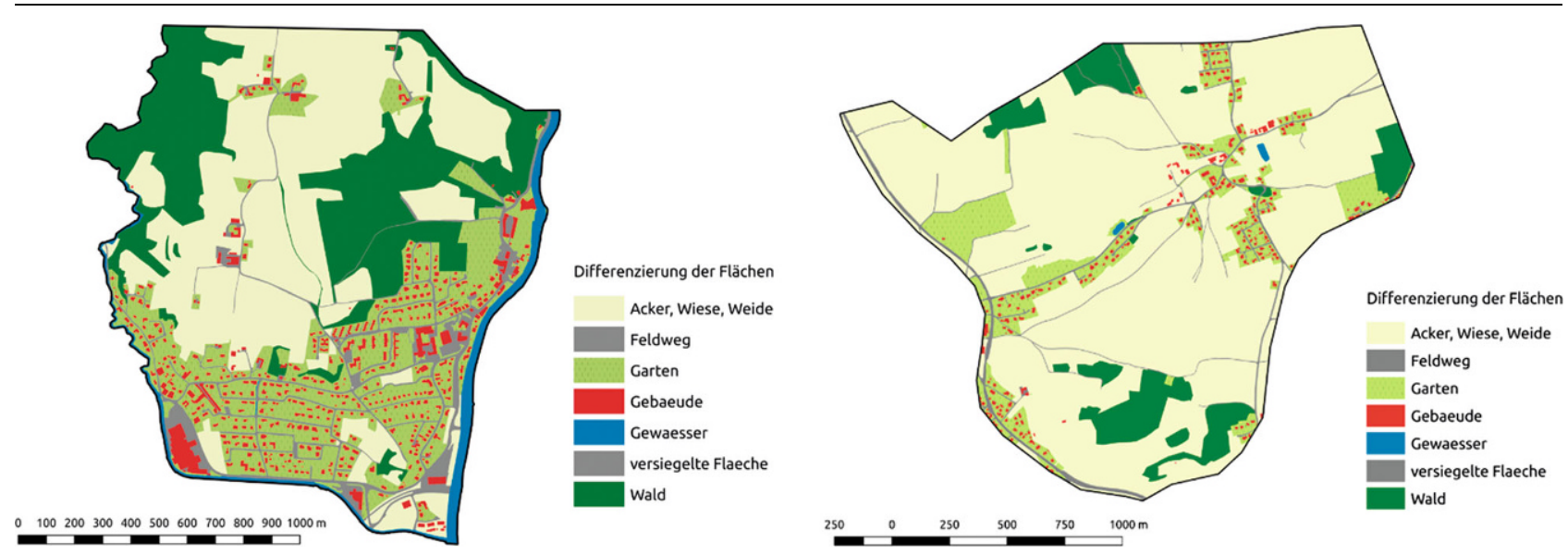

Abb. 2 Pilotgebiet Schwertberg, Poneggen (links) und Seewalchen, Kraims (rechts): Landnutzung

de wurden bei der Bearbeitung in einer Kategorie zusammengefasst. Dieser pragmatische Ansatz wurde gewählt, da Bestandsänderungen von Ackerflächen in Grünlandflächen grundsätzlich möglich sind und die jeweiligen Flächenanteile saisonal oder im mehrjährigen Verlauf variieren können.

\section{Methodik}

\subsection{Modelle und Anforderung}

Für die zweidimensionale hydrodynamische numerische Modellierung von Oberflächenabflüssen gibt es mittlerweile eine wachsende Anzahl von Softwarepaketen. Die verfügbaren Modelle unterscheiden sich dabei hinsichtlich der verwendeten hydrodynamischen Grundgleichungen (vollständige Flachwassergleichungen, vereinfachte hydraulische Berechnungsansätze), der verwendeten Rechennetzgeometrie (z.B. unstrukturierte Netze, rasterbasiert) und ihrer Lizenzierung (opensource, freeware, kommerziell).

Für die Gefährdungsanalyse von Starkregenereignissen bieten die unterschiedlichen Softwareprodukte zusätzlich verschiedene Funktionalitäten, wie z.B. die Möglichkeit der Berücksichtigung von Niederschlag als Randbedingung, die mögliche Kopplung an Modelle zur Berechnung der Kanalisation oder Funktionalitäten zur Modellierung von Abflussentstehungsprozessen.

Im Projekt RAINMAN wurden für drei Softwarepakete (Hydro_AS-2D, TELEMAC2D, FloodArea) ausgewählte Benchmark-Beispiele (vgl. Neelz und Pender 2013) nachgerechnet. Für die Softwareprodukte HEC RAS und JFlow lagen die Ergebnisse der Benchmark-
Tests bereits in Neelz und Pender (2013) bzw. US Army Corps of Engineers (2018) vor. Alle untersuchten Modelle sind in der Lage, die ausgewählten BenchmarkBeispiele nachzubilden. Dabei können sich jedoch bei gleichen Anfangs- und Randbedingungen Unterschiede zwischen den Modellergebnissen ergeben, die in der Wasserspiegellage bis zu einigen $\mathrm{cm}$ ausmachen. $\mathrm{Zu}$ vergleichbaren Ergebnissen kommen auch Broich et al. (2018) bei ähnlichen Untersuchungen.

Für die praktische Eignung/Anwendbarkeit zur Modellierung von pluvialen Überflutungen sind neben der ausreichend genauen Abbildung hydrodynamischer Prozesse auch noch zusätzliche Kriterien ausschlaggebend:

Möglichkeit zur Eingabe des Niederschlages als Wasserquelle im Modell:

- Ist die Eingabe des Niederschlags zeitlich variabel möglich?

- Kann ein räumlich variabler/verteilter Niederschlag definiert werden?

Hydrologischer Verlustansatz/Hydrologisches Modell:

- Sind Abflussbildungsmodelle in der Software implementiert? Welcher Modellansatz?

- Sind alternative Möglichkeiten zur Berücksichtigung möglich (z. B. Knoten/zellweise Eingabe von Nettoregen als Quellterm im hydrodynamischen Modell möglich)?

Einbau hydraulisch relevanter Strukturen:

- Können Mauern, Durchlässe etc. im Modell berücksichtigt werden?

Im Hinblick auf die Modellierung von Oberflächenabflüssen infolge von Starkregen sollte insbesondere darauf ge- achtet werden, dass die verwendete Software in der Lage ist, räumlich und zeitlich variierende Niederschlagdaten als Randbedingung zu verarbeiten. Neben der Nachrechnung realer Ereignisse (in der Bemessung nicht zwingend erforderlich) kann dies auch genutzt werden, um die Abflussbildung (Verlustbzw. Infiltrationsansatz) unabhängig vom verwendeten Modell im Vorfeld zu rechnen. Das hydraulische Modell wird dann mit dem Nettoregen beaufschlagt.

In diesem Beitrag werden die durchgeführten Untersuchungen im Überblick erläutert. Sensitivitäten zur Abflussbildung wurden primär im Einzugsgebiet Poneggen/Schwertberg ermittelt. Dabei kam die Software Hydro_AS-2D (Hydrotec 2019) in Kombination mit dem SCS-CN-Verfahren (SCS 1972; Klar et al. 2014; Humer et al. 2015) zum Einsatz. Bei den Untersuchungen zur Abflusskonzentration wurden verschiedene 2D-hydraulische Modelle in den unterschiedlichen Piloteinzugsgebieten angewendet. Dabei wurden sowohl die beiden rasterbasierten 2DHN-Modelle JFLOW und FloodArea als auch das mit flexiblen Rechennetzen arbeitende Modell Hydro_AS-2D untersucht.

\subsection{Niederschlagsszenarien}

Untersuchungen zu abgelaufenen pluvialen Überflutungen in weiten Gebieten Oberösterreichs im Sommer 2016 sowie Erfahrungen aus anderen Regionen legen nahe, dass vor allem intensive Niederschläge mit kurzer Dauerstufe als Auslöser für Überflutungen durch oberflächlich abfließendes Wasser/Hangwasser fungieren (Achleitner 

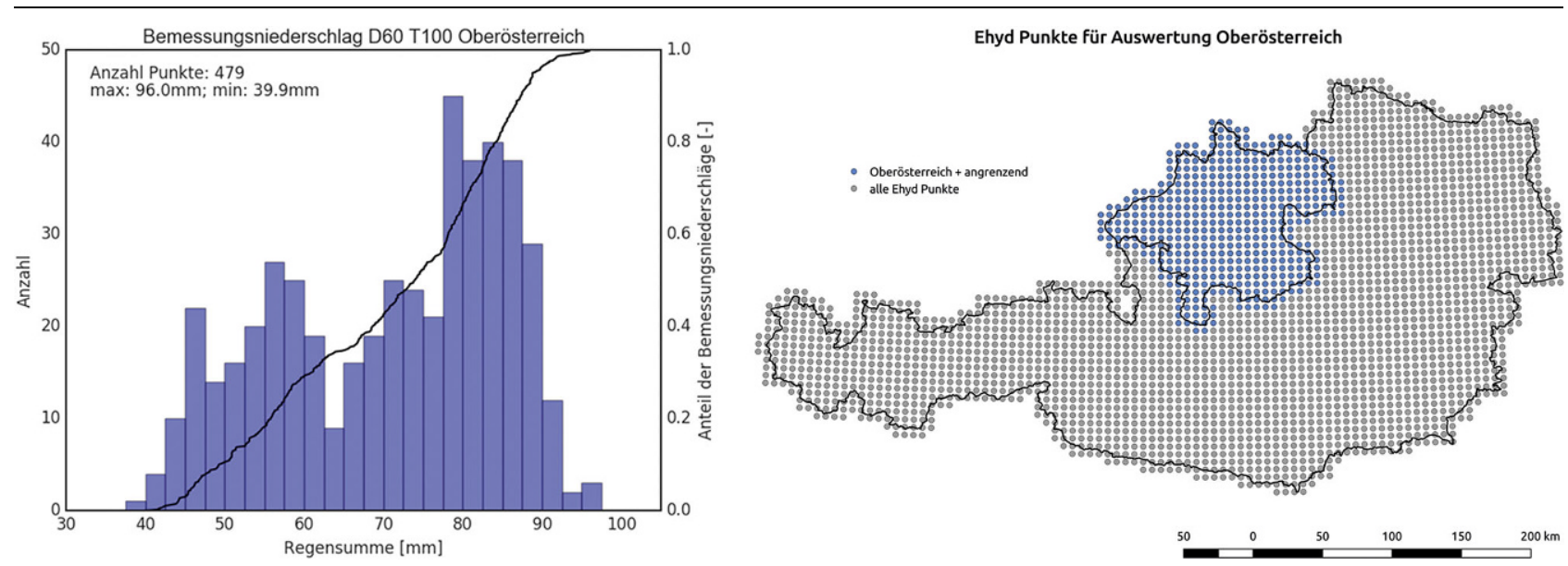

Abb. 3 Verteilung der 100-jährlichen eHYD-Bemessungsregen mit Dauer 60 min für Oberösterreich (links) und für die Auswertung herangezogene eHYD-Gitterpunkte (rechts)

et al. 2020a; Lumassegger et al. 2016a, 3.3 Abflussbildung b).

Als Richtwert für die Erstellung von Gefahrenhinweiskarten wird vom Amt der Oberösterreichischen Landesregierung die Verwendung eines 100-jährlichen eHYD-Bemessungsregens mit der Dauerstufe $30 \mathrm{~min}\left(\mathrm{~N}_{100,30}\right)$ empfohlen. Den gegenständlichen Berechnungen wurden 100-jährliche eHYDBemessungsregen mit der Dauerstufe 60 min $\left(\mathrm{N}_{100,60}\right)$ zugrunde gelegt. Abb. 3 zeigt die Verteilung der 100-jährlichen Regensummen mit Dauerstufe $60 \mathrm{~min}$ für Oberösterreich auf Basis der Bemessungsniederschläge 2009 (Daten von ehyd.gv.at vom Mai 2020; Weilguni 2009); die stündlichen Regensummen reichen dabei je nach geografischer Lage von etwa 40 bis $96 \mathrm{~mm}$.

In Untersuchungen zum Einfluss der Abflussbildung wurden jeweils die vor Ort gültigen Bemessungsniederschläge in den Piloteinzugsgebieten herangezogen. Für die Betrachtungen zur Abflusskonzentration wurde als Niederschlagsszenario generell ein Niederschlag mit einer Intensität von $62 \mathrm{~mm} / \mathrm{h}$ gewählt. Dies entspricht in etwa dem Mittel aus dem $\mathrm{N}_{100,60}$ (100-jähriger 60-minBemessungsniederschlag, eHYD-Datenstand 2009) der Pilotgebiete Seewalchen und Schwertberg. Auf individuelle (unterschiedliche) Regeneingangsdaten wurde bewusst verzichtet, um in den Sensitivitätsuntersuchungen gleiche Randbedingungen in beiden Gebieten zu nutzen. Für ausgewählte Szenarien wurden zusätzlich der maximale und der minimale $\mathrm{N}_{100,60}(96 \mathrm{~mm} / \mathrm{h}$ bzw. $40 \mathrm{~mm} / \mathrm{h}$ ) innerhalb Oberösterreichs verwendet (vgl. Abb. 3).
Die Abflussentstehung auf unterschiedlichen Flächen ist u.a. abhängig von Faktoren wie der aktuellen Vegetationsbedeckung, dem Versiegelungsgrad, der Landnutzung und den Bodeneigenschaften. Die meisten dieser Faktoren sind zeitlich variabel; in Abhängigkeit von den aktuell vorherrschenden Verhältnissen kann es bei vergleichbaren Niederschlägen zu unterschiedlich ausgeprägter Oberflächenabflussbildung kommen, und eine entsprechende Berücksichtigung dieser Faktoren in der Modellierung ist daher wichtig. In 2D-HN-Modellen, in denen räumlich und zeitlich variabler Niederschlag als Randbedingung möglich sind, können unabhängig vom implementierten Verlustansatz entsprechende Berechnungen im Pre-Processing durchgeführt werden; der vorab berechnete Nettoregen für unterschiedliche Zeitschritte kann dann für jedes Element des Rechennetzes/-rasters als Randbedingung definiert werden. Aus praktischer Sicht stellt sich neben der Wahl des entsprechenden Verlustmodells aber vor allem die Frage nach dessen Parametrisierung.

Im Rahmen der Projekte SAFFER-CC (Achleitner et al. 2019) und RAINMAN (Achleitner et al. 2020b, c) wurden 2015, 2016 und 2018 in Schwertberg, Seewalchen und Leonding Beregnungsversuche durchgeführt, um das $\mathrm{Ab}$ flussverhalten auf der Plotskale mit Testflächen von $5 \times 10 \mathrm{~m}$ bzw. $5 \times 16 \mathrm{~m} \mathrm{zu}$ erfassen. Dabei wurden unterschiedliche Landnutzungstypen wie Mais, Soja, Zuckerrüben, Getreide und Grünland mit mobilen Beregnungsanlagen be- regnet. Die Beregnung wurde jeweils mit $100 \mathrm{~mm} / \mathrm{h}$ und einer Versuchsdauer von $1 \mathrm{~h}$ durchgeführt. Nachfolgend wurden einzelne Flächen mit zeitlich variablem Niederschlag und Intensitätsspitzen bis $180 \mathrm{~mm} / \mathrm{h}$ beaufschlagt (Abb. 4).

Neben der Messung des Oberflächenabflusses, wurde bei diesen Experimenten auch die Bodenfeuchte in verschieden Tiefen erfasst. Mit unterschiedlichen empirischen und physikalisch-basierten Modellansätzen unterschiedlicher Komplexität können die maßgeblichen Abflussbildungsprozesse beschrieben werden. Die in den unterschiedlichen Softwarepaketen implementierten Ansätze variieren jedoch. In den hier durchgeführten Untersuchungen wurde das SCS-CN-Verfahren verwendet. Die Parameterwahl erfolgte spezifisch für unterschiedliche Standorte, Landnutzungen und Vorbedingungen, anhand der Beregnungsergebnisse. Dazu wurde ein CN-Modell jeweils an den aus den Versuchen gewonnenen Daten kalibriert, sodass der finale Spitzenabflussbeiwert und der zeitliche Verlauf des Oberflächenabflusses bestmöglich abgebildet werden. Diese CN-Werte für unterschiedliche Standorte, Landnutzungen und Vorbedingungen bildeten die Grundlage für die Parametrisierung des gekoppelten hydrologisch-2D-hydraulischen Modells.

In den Sensitivitätsuntersuchungen zur Abflussbildung wurden diese Parametrisierungen genutzt, um entsprechende Extremszenarien $\mathrm{zu}$ entwickeln. Alle in den Einzugsgebieten befindlichen landwirtschaftlichen Flächen wurden dabei auf einheitliche 


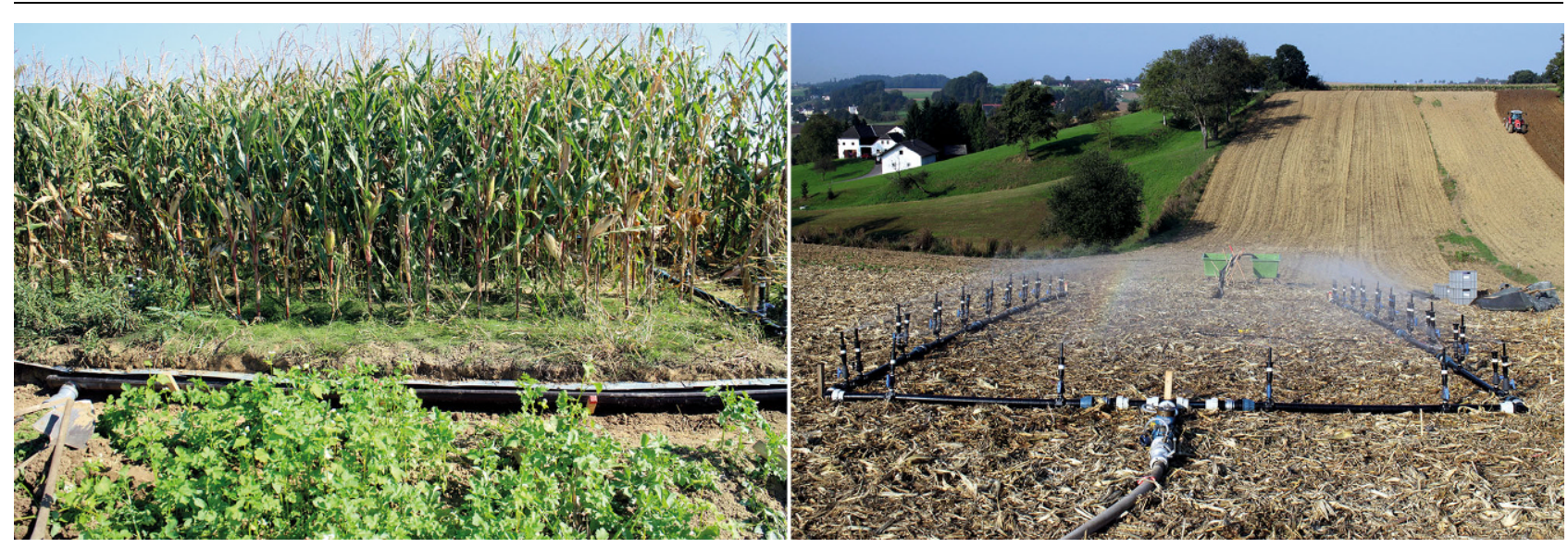

Abb. 4 Beregnungsexperimente Schwertberg 2015, Maisfeld vor und nach Ernte

Landnutzungen und Vorbedingungen gesetzt.

\subsection{Abflusskonzentration}

Neben den Abflussbildungsprozessen wurde im Zuge des Projekts RAINMAN vor allem die Nachbildung von Abflusskonzentrationsprozessen in der 2DHN-Modellierung näher untersucht. Insbesondere wurden dabei Aspekte der Modellerstellung und Parametrisierung im Zuge von Sensitivitätsanalysen beleuchtet. In Bezug auf die Modellbzw. Rechennetzerstellung wurde in einem ersten Schritt der Einfluss unterschiedlicher Rechennetz- bzw. Rasterauflösungen auf die modellierten max. Wassertiefen untersucht. Nachfolgend wurden Untersuchungen zur Berücksichtigung verschiedener potenziell hydraulisch relevanter Strukturen (Gartenmauern, Durchlässe) im Rechennetz durchgeführt und deren Einfluss auf die Modellergebnisse betrachtet. In Bezug auf die Modellparametrisierung wurden Varianten mit unterschiedlichen Fließwiderstandsbeiwerten nach Manning/Strickler $\left(\mathrm{k}_{\mathrm{st}}\right)$ untersucht und ausgewertet.

Um diese Effekte entkoppelt von unterschiedlichen, räumlich verteilten Abflussbildungsprozessen $\mathrm{zu}$ untersuchen, blieben diese unberücksichtigt es wurde ohne Versickerungsanteile gerechnet. Die gesamte Simulationsdauer wurde mit $120 \mathrm{~min}$ festgelegt, um nach dem auftretenden 60-min-Niederschlag etwaige verzögerte ablaufende Wellen mit zusätzlich 60 min Nachlaufzeit abzudecken.

\subsubsection{Modellauflösung}

Bei den Untersuchungen hinsichtlich Modellauflösung muss grundsätzlich zwischen Rastermodellen und unstrukturierten/flexiblen Rechennetzen unterschieden werden. Während bei rasterbasierten Modellen die Auflösung des digitalen Höhenmodells (DGMs) auch der des Rechennetzes entspricht, ist die Auflösung des Rechennetzes bei Modellen, die mit flexiblen Rechennetzen arbeiten, grundsätzlich von der des DGMs entkoppelt.

Bei Rastermodellen verschlechtert sich bei zunehmender Vergröberung der Auflösung gleichzeitig die Abgrenzung und Lagegenauigkeit von Bereichen mit unterschiedlichen hydrologischen und hydraulischen Eigenschaften. Bei flexiblen Rechennetzen ermöglichen Bruchkanten eine lagetreue, von der Netzauflösung unabhängige Abbildung von z.B. Gebäudegeometrien, Geländekanten, Verkehrsflächen, unterschiedlichen Landnutzungen usw.

Um den Einfluss unterschiedlicher Netzauflösungen auf modellierte Oberflächenabflusstiefen abzuschätzen, wurden in zwei Pilotgebieten Simulationen mit Hydro_AS-2D (unstrukturiertes Rechennetz) und FloodArea (rasterbasiertes Modell) mit variierenden Netz- bzw. Rasterauflösungen durchgeführt.

Während für die Simulationen mit Hydro_AS-2D Gebäudeflächen aus dem Rechennetz entfernt wurden, wurden für die Berechnungen mit FloodArea die Gebäudeflächen im DGM pauschal um $7 \mathrm{~m}$ erhöht. In beiden Herangehensweisen wurden damit die entsprechenden Flächen als nicht überströmbar modelliert.
Im Zuge der Simulationen mit flexiblem Netz (Hydro_AS-2D) wurden zudem unterschiedliche Variationen der Netzauflösung untersucht, bei denen beispielsweise eine hohe Netzauflösung im Siedlungsbereich mit einer gröberen Diskretisierung des Vorlandes kombiniert wurde.

\subsubsection{Parametrisierung der Rauheit (Strickler Beiwerte)}

In den meisten gängigen 2D-HN-Softwarepaketen, die in der Praxis für die Modellierung von Oberflächenabflüssen verwendet werden, wird der Fließwiderstand unterschiedlicher Oberflächen über entsprechende Rauheitsbeiwerte (meist Manning-( $n$ ) bzw. Stricklerbeiwert $\left(\mathrm{k}_{\mathrm{St}}\right)$ ) definiert, die als Randbedingung in die Modellierung eingehen. Für unterschiedliche Oberflächenklassen (zumeist angelehnt an die Landnutzung und Landbedeckung) werden dabei verschiedene Fließwiderstände definiert. In Ermangelung gemessener Abflüsse in den Untersuchungsgebieten, die für eine Kalibrierung der Fließwiderstände herangezogen werden könnten, wird in vielen Studien auf Literaturwerte zurückgegriffen.

Bernet et al. (2018) verwenden beispielsweise für die Modellierung von Oberflächenabflüssen in Schweizerischen Einzugsgebieten mit FloodArea und FLO-2D Werte für hydrologische Fließoberflächen aus McCuen (2016), auch in Maniak (2010) finden sich ähnliche Angaben. Tyrna et al. (2017) verwenden für die Rauheitsparametrisierung von Modellrechnungen mit FloodArea Werte aus Literaturtabellen (Engman 1986 und Chow 1959). Kipfer 


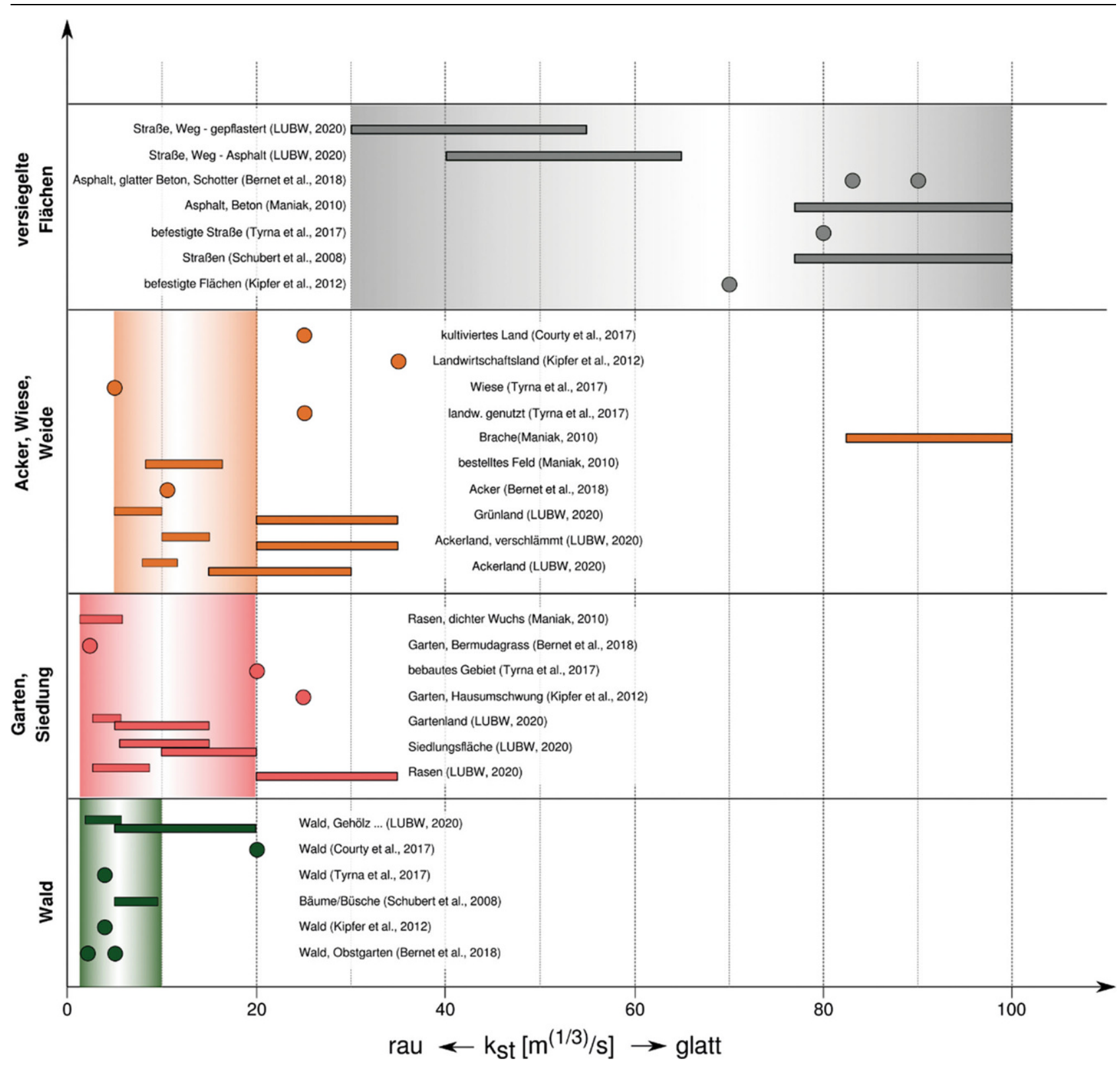

Abb. 5 Rauheitsbeiwerte nach Strickler $\left(k_{s t}\right)$ aus unterschiedlichen Quellen. Farblich hinterlegt sind die in der Studie betrachteten Wertebereiche

et al. (2012) geben auch $\mathrm{k}_{\mathrm{st}}$-Werte an, die für die Modellierung von Oberflächenabflüssen in der Schweiz mit der Software FloodArea herangezogen wurden. Ähnliche Tabellen finden sich u.a. in Schubert et al. (2008) oder Courty et al. (2017). Neben der Oberflächenbeschaffenheit ist der Fließwiderstand einer Fläche auch von der Fließtiefe abhängig. Dieser Abhängigkeit trägt eine wachsende Anzahl von Software-Paketen Rechnung, indem sie die Definition fließtiefenabhängiger Fließwiderstände erlauben. Für die Modellierung pluvialer Überflutungen mit fließtiefenabhän- gigen Rauheiten gibt es aus Sicht der Autoren bislang allerdings nur wenige Erfahrungen. Einen ersten Ansatz mit Fokus auf die praktische Modellanwendung findet sich in LUBW (2020). Darin werden Rauheitsbeiwerte für Dünnfilmabfluss (bis $2 \mathrm{~cm}$ ) und Rauheitsbeiwerte für Fließtiefen $>10 \mathrm{~cm}$ vorgeschlagen. Für Abflüsse mit geringen Wassertiefen liefern auch Markart et al. (2005) und Kohl (2011) wertvolle Hinweise. Ein Überblick der Einzelwerte und Bandbreiten für $\mathrm{k}_{\mathrm{st}}$-Werte aus der Literatur ist in Abb. 5 zusammengestellt.
Im Zuge der Untersuchungen wurden, in Anlehnung an bereits publizierten Wertebereiche aus vorangegangenen Studien, für die in den Untersuchungen berücksichtigten Nutzungsklassen drei unterschiedliche Rauheitsparametrisierungen untersucht. Eine „mittlere“ Basis-Parametrisierung (hier als Referenz bezeichnet) sowie eine entsprechend raue und glatte Parametervariante wurden mit Hydro_AS-2D und FloodArea untersucht. Die in Abb. 5 farblich schattierten Bereiche decken die modellierten Szenarien $(\mathrm{min} / \mathrm{max}$ 
Tab. 1 Übersicht Szenarien für Durchlässe. Die Berechnungen wurden in Hydro_AS-2D und JFlow durchgeführt

$\begin{array}{ll}\text { Regenszenario } & \text { Durchlass-Dimensionen }(\mathrm{DN}) \\ 62 \mathrm{~mm} / \mathrm{h}, 1 \mathrm{~h}, \text { Blockregen } & {[100,200,300,400,500,600,800,1000]} \\ 40 \mathrm{~mm} / \mathrm{h}, 1 \mathrm{~h}, \text { Blockregen } & {[300,500,1000]} \\ 96 \mathrm{~mm} / \mathrm{h}, 1 \mathrm{~h}, \text { Blockregen } & {[300,500,1000]}\end{array}$

Tab. 2 Übersicht Szenarien für Mauern. Die Berechnungen wurden in FloodArea und Hydro_AS-2D durchgeführt

\begin{tabular}{|l|l|l|}
\hline Regenszenario & Mauerhöhen offen $[\mathrm{cm}]$ & Mauerhöhen geschlossen [cm] \\
\hline $62 \mathrm{~mm} / \mathrm{h}, 1 \mathrm{~h}$, Blockregen & {$[5,10,25,50,100,200]$} & {$[5,10,25,50,100,200]$} \\
\hline $40 \mathrm{~mm} / \mathrm{h}, 1 \mathrm{~h}$, Blockregen & {$[10,25,100]$} & {$[10,25,100]$} \\
\hline $96 \mathrm{~mm} / \mathrm{h}, 1 \mathrm{~h}$, Blockregen & {$[10,25,100]$} & {$[10,25,100]$} \\
\hline
\end{tabular}

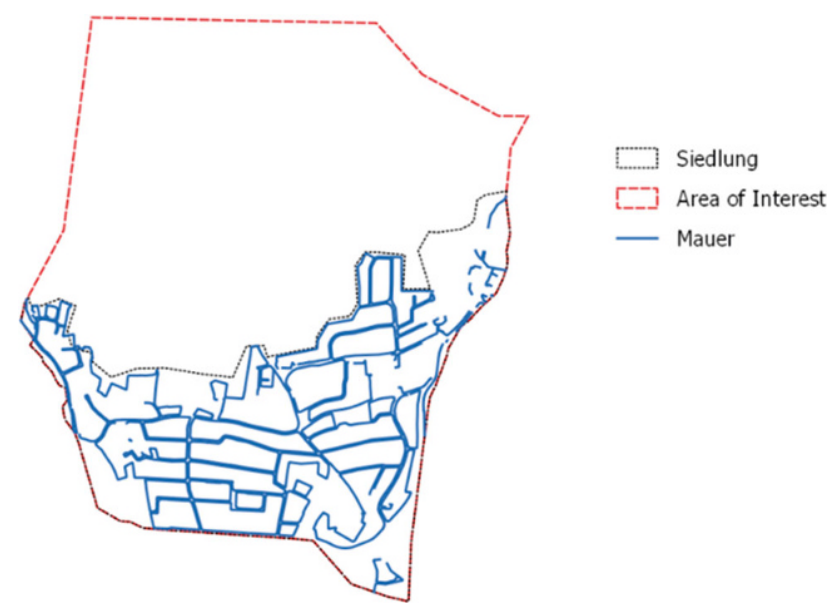

Abb. 6 Lage der Mauern für die Variantenstudie mit unterschiedlichen Mauerhöhen im Einzugsgebiet Poneggen/Schwertberg
28.09.2016

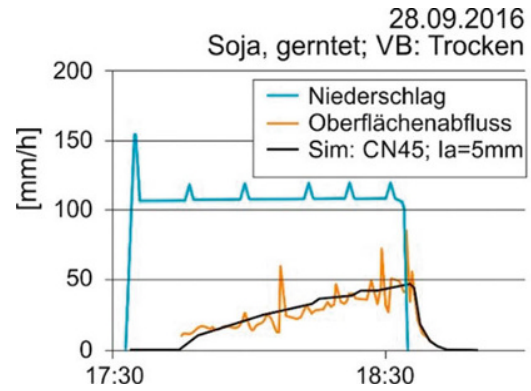

30.09.2016

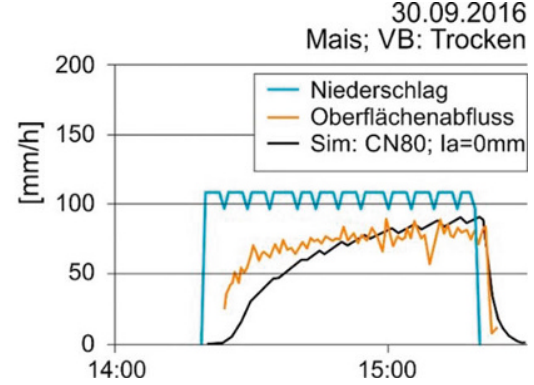

Abb. 7 Ergebnisse der Beregnungsversuche auf Anbauflächen für Soja (geerntet) und Mais mit trockenen Vorbedingungen(VB). Modellierte Abflussbildung (schwarz) mit CNVerfahren

Wert) sowie den Mittelwerten als Referenzszenario ab.

\subsubsection{Erhebung und Einbau von Durchlässen}

Bei der Modellerstellung stellt sich in der Praxis häufig die Frage, inwieweit Strukturen wie Unterführungen, Verdolungen oder Durchlässe im Modell integriert werden müssen, bzw. in wel-
Die modellierten Durchmesser wurden zwischen DN 100 bis DN 1000 variiert. Rechenläufe mit Berücksichtigung von Durchlässen wurden im Zuge der Pilotstudie mit Hydro_AS-2D und JFlow durchgeführt. Um den Einfluss der Berücksichtigung der Durchlässe mit unterschiedlichen Größen im Modell einschätzen zu können, erfolgte ein Vergleich mit einem Referenzszenario („keine Durchlässe“). Zusätzlich zu den Varianten mit stündlichen Regensummen von $62 \mathrm{~mm}$ wurden auch noch einzelne Vergleichsrechnungen mit 40 und $96 \mathrm{~mm}$ Stundenniederschlagssummen durchgeführt (vgl. Abschn. 3.2). Tab. 1 gibt einen Überblick.

\subsubsection{Lokale Fließhindernisse und Mauern}

Im Ereignisfall können im Siedlungsbereich Grundstückseinfriedungen in Form von Zäunen, Mauern oder Hecken die lokalen Strömungsverhältnisse beeinflussen. Da die hydraulische Wirksamkeit von teilweise durchlässigen Abgrenzungen wie Zäunen oder dichter Vegetation im Ereignisfall nur schwer abschätzbar ist (z. B. Verklausung, unterschiedliche Bautypen etc.) beschränken sich die hier vorgestellten Untersuchungen auf die Sonderfälle geschlossene Mauer und Mauer mit Öffnung (z.B. Hauseinfahrten, Gartentor etc.).

Die Wirkung von Mauern wurde anhand eines generischen Beispiels, wieder im Untersuchungsgebiet Schwertberg, untersucht. Entlang der straßenseitigen Grundstücksgrenzen wurden im Siedlungsraum fiktive Mauern in den Rechennetzen implementiert. Insgesamt wurden Mauern in der kumulierten Länge von ca. $22.700 \mathrm{~m}$ in die Rechennetze bzw. -raster eingebaut (Abb. 6). Für die Variante geschlossene Mauer wurde eine vollständige Umrandung der Grundstücke mit Mauern angenommen, für die Variante mit offenen Mauern wurde die Lage und Länge der Öffnungen anhand eines Orthofotos für Hauseinfahrten und Eingänge bestimmt. Der Anteil der Öffnungen an der Gesamtlänge der eingebauten Mauern beträgt in diesen Varianten ca. $14 \%$.

Um den Einfluss verschieden hoher Mauern mit und ohne Öffnungen zu untersuchen, wurden unterschiedliche Varianten mit Hydro_AS-2D und FloodArea betrachtet. Tab. 2 gibt einen Überblick über die durchgeführten Re- 

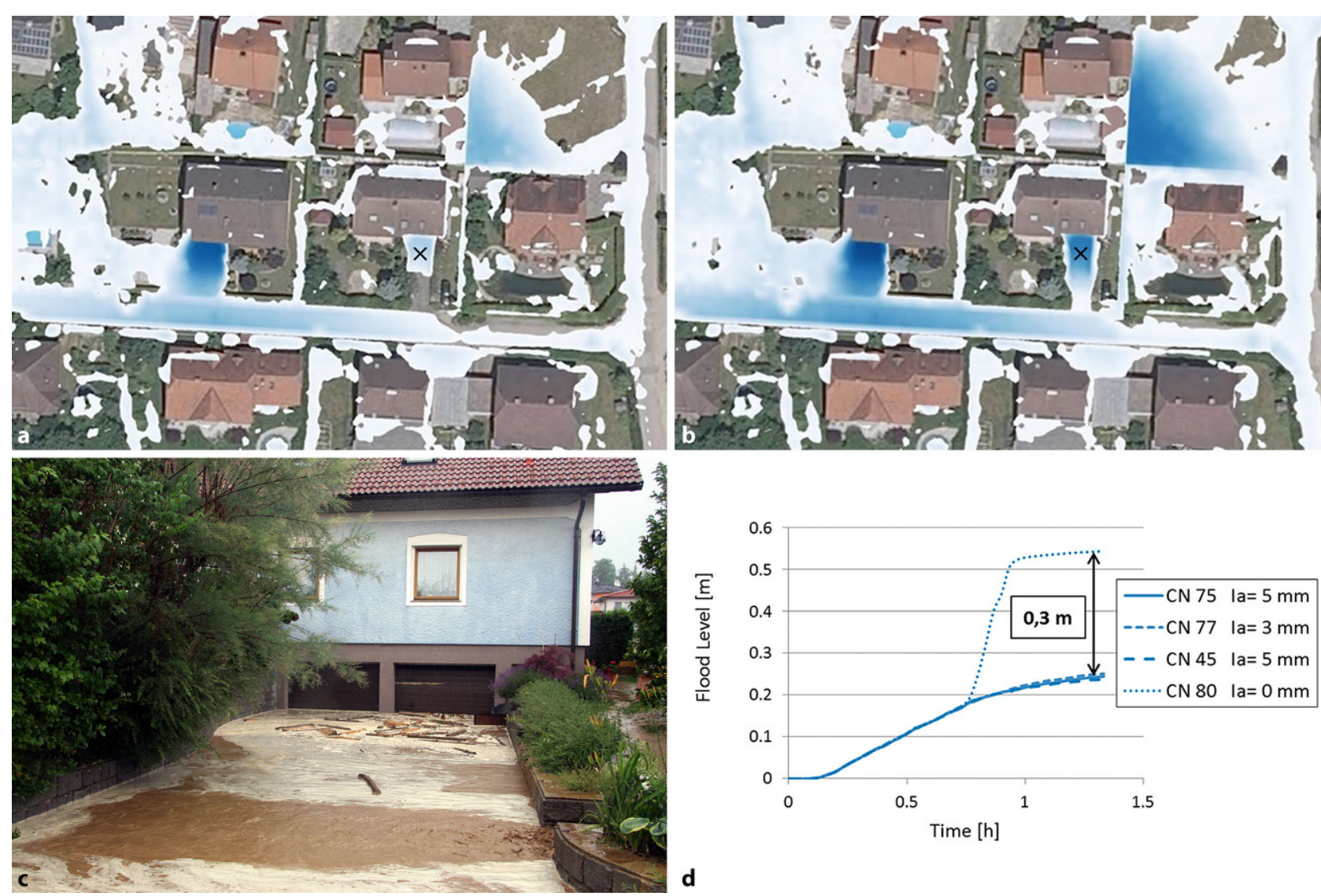

Abb. 8 Vergleich der Einstauhöhen und Überflutungsflächen in Poneggen/Schwertberg (Teilbereich) bei Annahme unterschiedlicher landwirtschaftlichen Nutzungen; Überflutungsflächen a Soja (geerntet) - CN 45, b Mais - CN 80
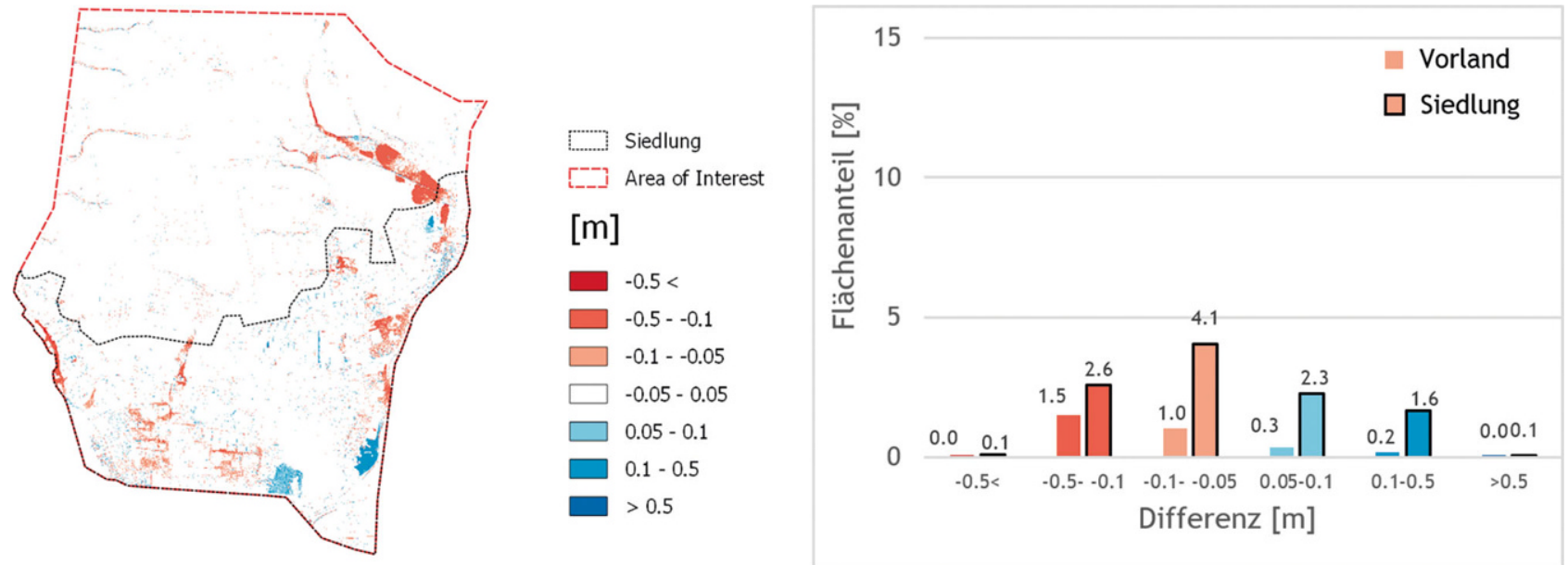

Abb. 9 Differenz der max. Wasserstände (Pilotgebiet Schwertberg); Simulationen mit FloodArea basierend auf 1-m- bzw. 0,5-mRaster/DGM

chenläufe. Wie im vorangegangenen Abschnitt wurde auch hier ein einstündiges Regenereignis mit räumlich und zeitlich konstanter Intensität zugrunde gelegt, Infiltrationsverluste wurden nicht berücksichtigt.

\section{Ergebnisse}

4.1 Abflussbildung

Die Sensitivität der landwirtschaftlichen Flächenbewirtschaftung auf die Entwicklung von Überschwemmun- gen wird am Beispiel von Poneggen/ Schwertberg gezeigt. Die verwendeten $\mathrm{CN}$-Werte wurden anhand der vorgestellten Niederschlagsversuche kalibriert, wobei nach Standort, Landnutzung und Vorbedingung unterschieden wurde. Exemplarisch sind in Abb. 7 


\section{Originalarbeit}

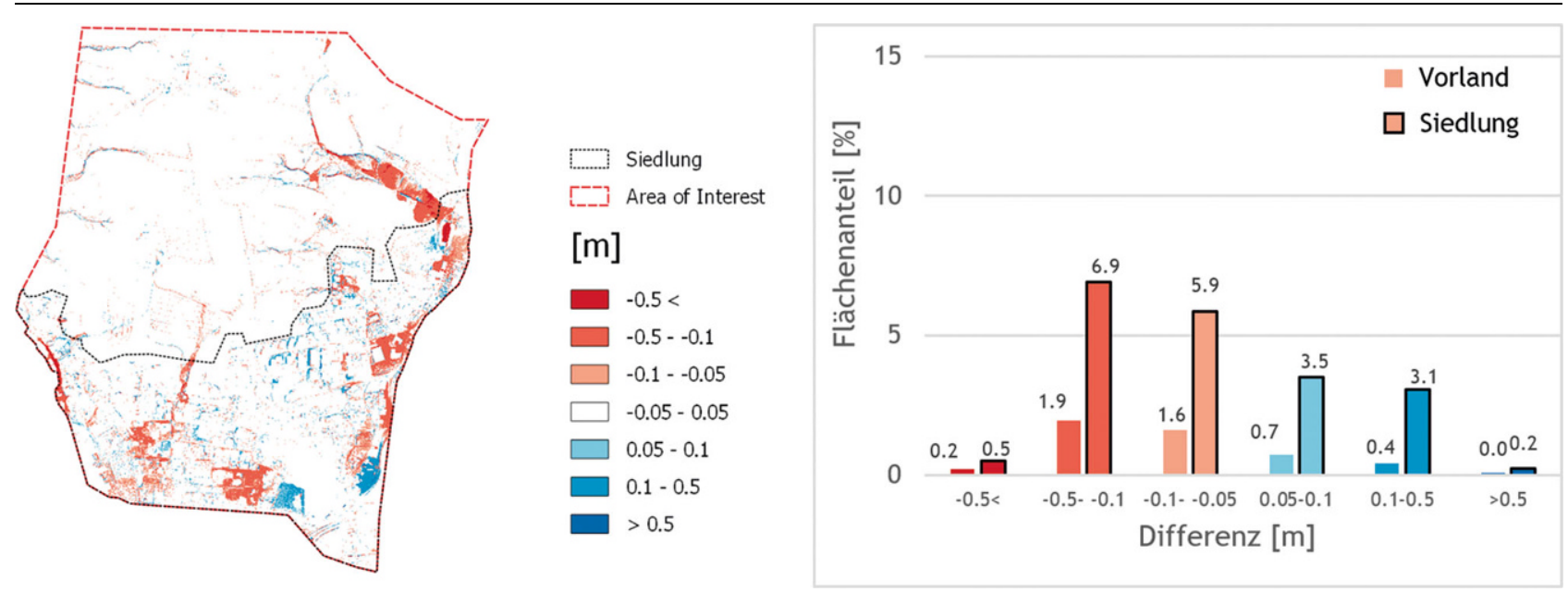

Abb. 10 Differenz der max. Wasserstände (Pilotgebiet Schwertberg); Simulationen mit FloodArea basierend auf 2,5-m- bzw. 0,5-mRaster/DGM
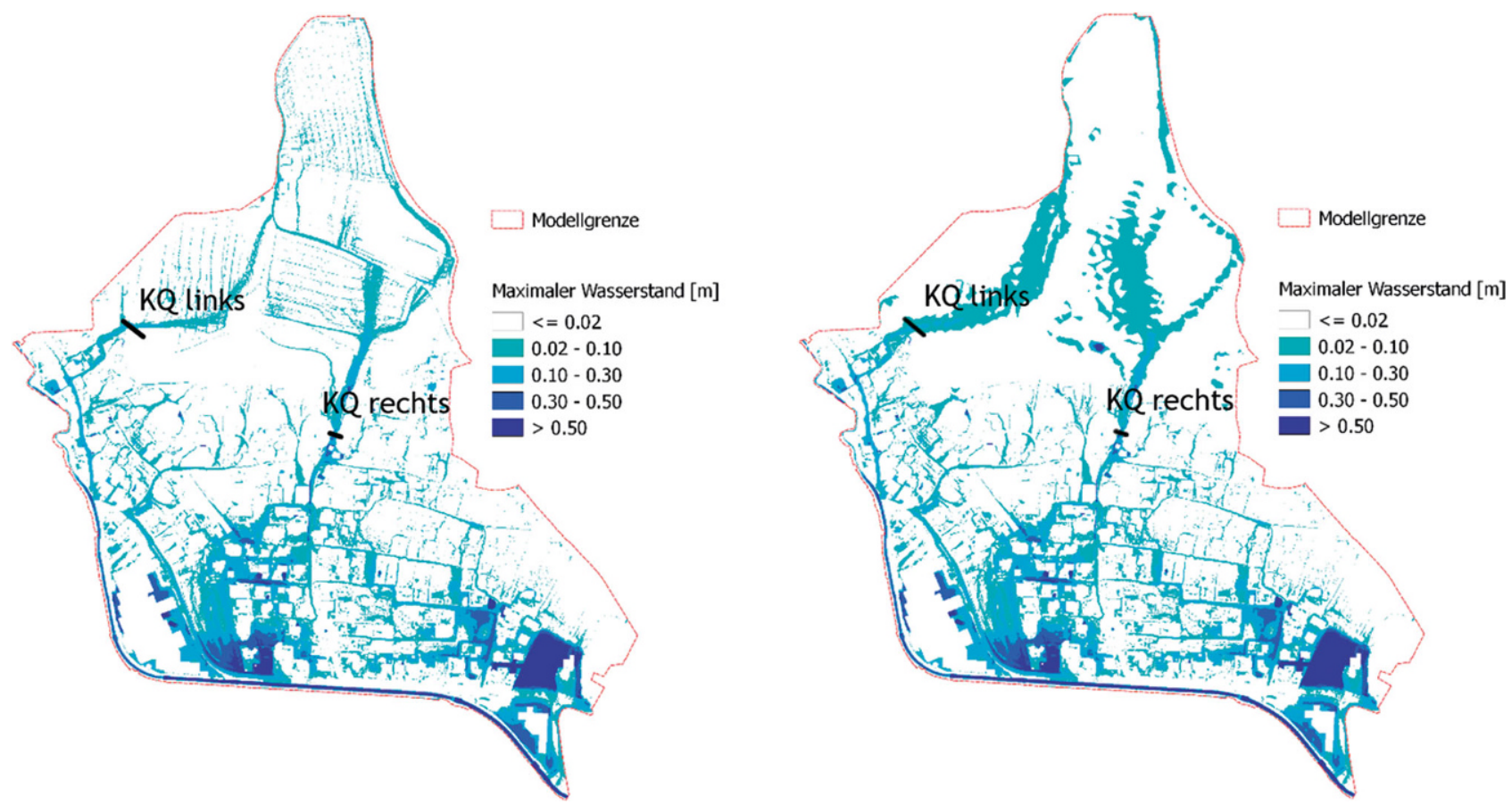

Abb. 11 Maximale modellierte Wasserstände für ein Szenario mit durchgehend feiner Netzauflösung von 0,5 $\mathrm{m}$ (links) und variabler Netzauflösung (rechts, 0,5 m im Siedlungsbereich, $10 \mathrm{~m}$ außerhalb) mit Hydro_AS-2D. Die Kontrollquerschnitte liegen an den Haupteintrittspunkten von Oberflächenwasser in den Siedlungsraum

die Verläufe des Niederschlags und Oberflächenabflusses bei Beregnungsversuchen auf Anbauflächen für Soja (geerntet) und Mais dargestellt.

In der Kalibrierung wurde darauf geachtet, dass sowohl der zeitliche Verlauf der Oberflächenabflussentwicklung als auch der Spitzenabflussbeiwert bei Beendigung des Beregnungsversuchs möglichst gut abgebildet werden. Für Szenarien unterschiedlicher Landnutzung ergeben sich dabei durchaus signifikante Unterschiede in den Überflutungsflächen als auch Einstauhöhen. Abb. 8 zeigt den Ausschnitt eines Simulationsergebnisses in Schwertberg/ Poneggen, wobei die Landnutzung von Agrarflächen im Gebiet einer kompletten Nutzung durch Soja (geerntet) bzw. Mais entspricht. Die Simulationen wurden jeweils mit dem lokal gültigen Bemessungsniederschlag (N100, D60, Stand 2009) durchgeführt, wobei dieser vereinfacht als räumlich gleichverteilter Blockregen angesetzt wurde.

\subsection{Abflusskonzentration}

Die Auswertungen der durchgeführten Sensitivitätsanalysen wurden separat für unterschiedliche Teile der Untersuchungsgebiete durchgeführt. Dabei wurden die Einzugsgebiete in die $\mathrm{Ka}$ tegorien Siedlungsgebiet und Vorland unterteilt. Die als Siedlungsgebiet de- 

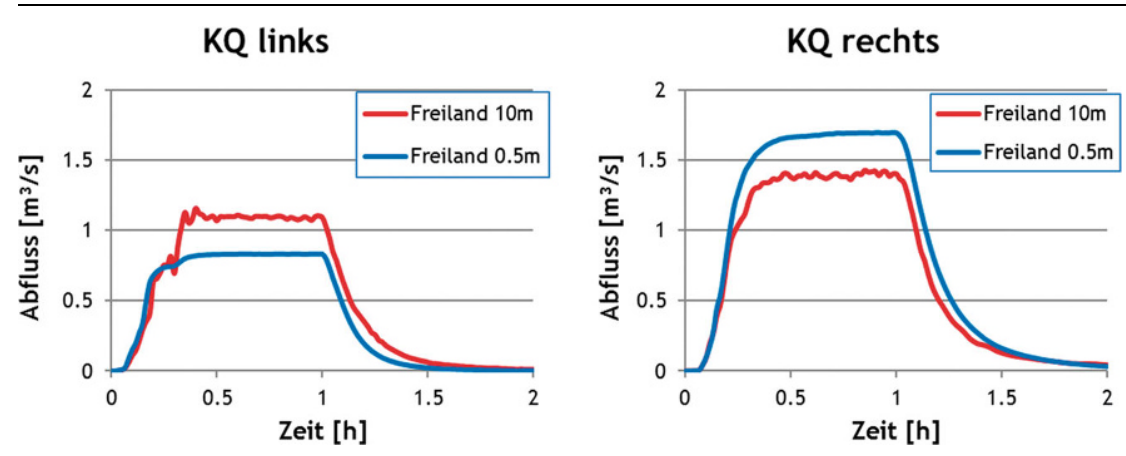

Abb. 12 Abflussganglinien für die beiden Kontrollquerschnitte aus Abb. 11. Eine Vergröberung der Netzauflösung außerhalb des Siedlungsbereiches von 0,5 auf $10 \mathrm{~m}$ führt zueinerVerlagerung derAbflussanteile, die durch die beiden Kontrollquerschnitte in den Siedlungsraum gelangen
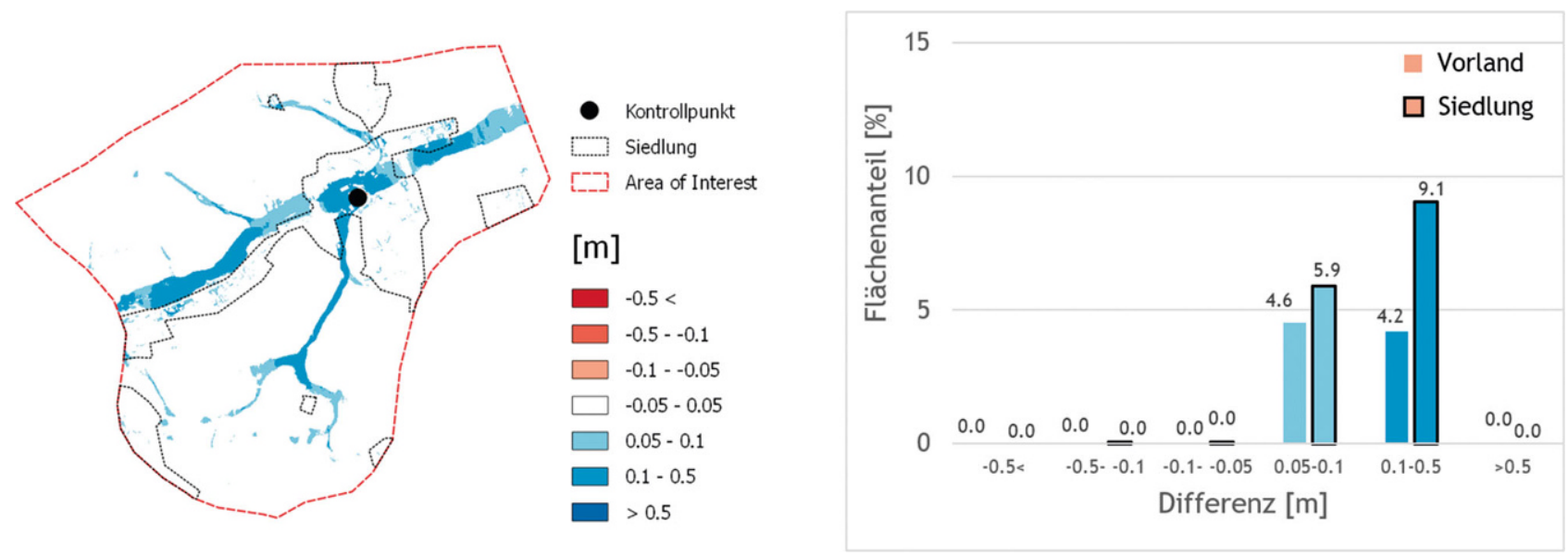

Abb. 13 Differenzraster Seewalchen, berechnet aus maximal modellierte Wassertiefe Szenario rau-Referenz mit Säulendiagrammen berechnet mit Hydro_AS-2D. DieFlächenanteilewurden separat fürVorland-und Siedlungsflächeausgewertet. Differenzenkleiner $\pm 5 \mathrm{~cm}$ werden im Säulendiagramm nicht dargestellt
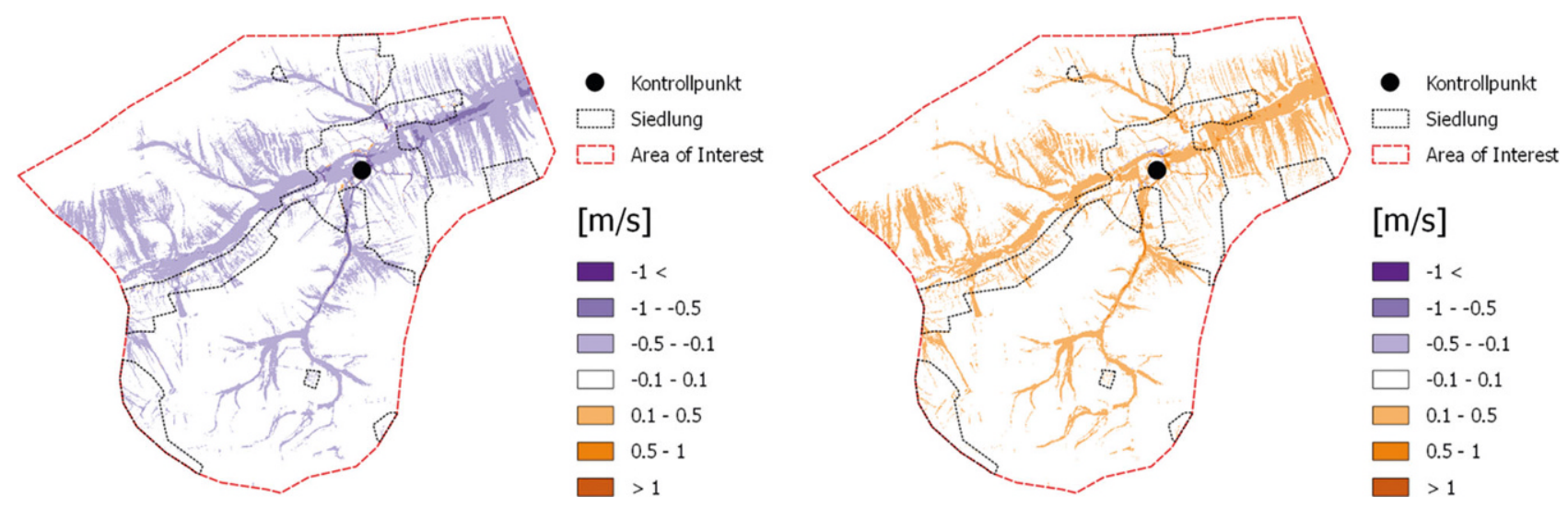

Abb. 14 Differenzraster Seewalchen berechnet aus maximale Geschwindigkeit Szenario rau-Referenz (links) bzw. Szenario glattReferenz (rechts) berechnet mit Hydro_AS-2D 


\section{Originalarbeit}
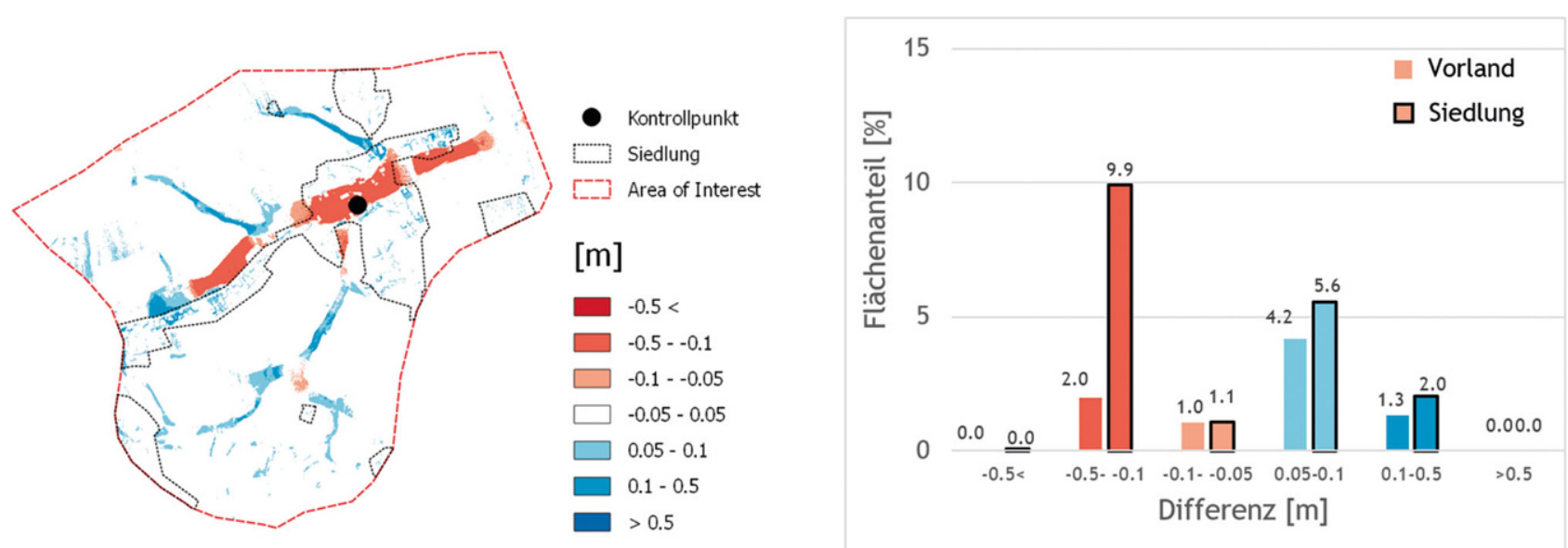

Abb. 15 Differenzraster Seewalchen, berechnet aus maximal modellierte Wassertiefe Szenario rau-Referenz mit Säulendiagrammen berechnet mit FloodArea. Die Flächenanteile wurden separat für Vorland- und Siedlungsfläche ausgewertet. Differenzen kleiner $\pm 5 \mathrm{~cm}$ werden im Säulendiagramm nicht dargestellt
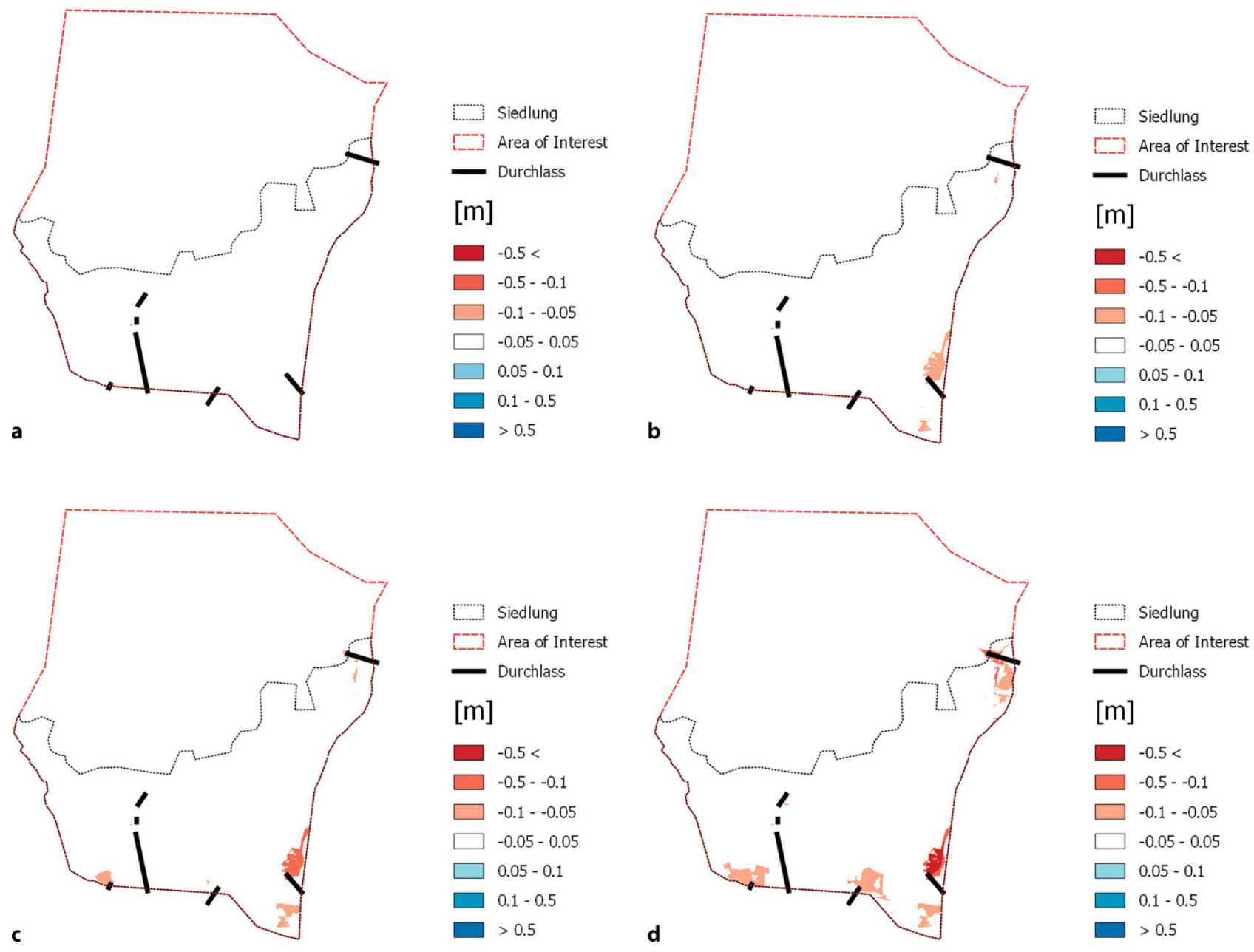

Abb. 16 Vergleich der maximalen modellierten Fließtiefen für Varianten mit unterschiedlichen Durchlassgrößen im Vergleich zum Referenzszenario ohne Durchlässe; Regenszenario $62 \mathrm{~mm} / \mathrm{h}, 1 \mathrm{~h}$. Die Lage der Durchlässe im Modell ist in Schwarz dargestellt. a Differenzraster DN 400 mm, b Differenzraster DN 500 mm, c Differenzraster DN 600 mm, d Differenzraster DN 1000 mm 


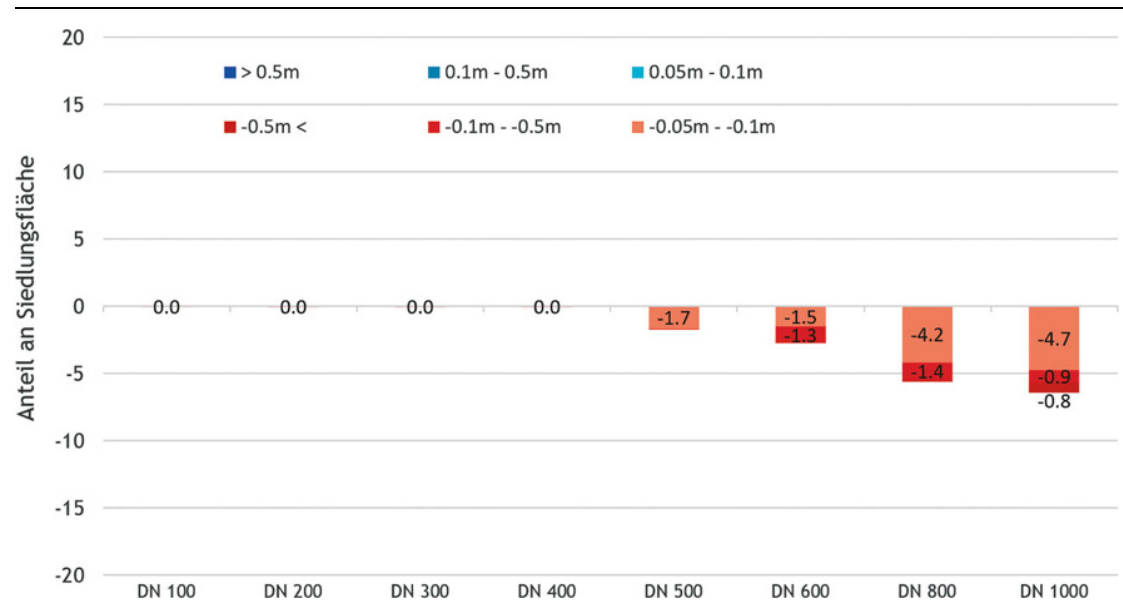

Abb. 17 Auswertung der Siedlungsflächen mit Abweichungen größer als $\pm 5 \mathrm{~cm}$ gegenüber dem Referenzszenario ohne Durchlass in Abhängigkeit vom Nenndurchmesser für Blockregen $62 \mathrm{~mm} / \mathrm{h}$ in Schwertberg berechnet mit Hydro_AS-2D. Ein negatives Vorzeichen bedeutet, dass die maximaleFließtiefe dieser Flächen im Szenario niedriger ist als im Referenzszenario

finierten Bereiche umfassen die mehr oder weniger geschlossenen Siedlungsbereiche in den Einzugsgebieten, die Kategorie Vorland beinhaltet alle Bereiche außerhalb der Siedlungsbereiche und setzt sich in den Untersuchungsgebieten hauptsächlich aus Agrar- und Forstflächen in den höher gelegenen Einzugsgebietsbereichen zusammen. Für diese Kategorien ergibt sich ein Flächenverhältnis von $82 \%$ Vorland zu $18 \%$ Siedlung in Seewalchen, und von 59,5\% Vorland zu 40,5\% Siedlung in Schwertberg.

\subsubsection{Modellauflösung}

Exemplarisch für die Untersuchungen der Netzauflösung bei rasterbasierten Modellen wird das Beispiel Schwertberg mit Netzauflösungen von $1 \mathrm{~m}$ bzw. $2.5 \mathrm{~m}$ vorgestellt. Die in Abb. 9 und 10 dargestellten Ergebnisse zeigen die Differenzen der maximalen simulierten Wassertiefen für die Modellläufe mit unterschiedlicher Auflösung (1 m, 2,5 m) im Vergleich zu den Ergebnissen des Referenzszenarios (Berechnung mit $0,5 \mathrm{~m}$ Rasterauflösung). Über- oder unterschätzte Wasserstände sind dabei als Flächen in Blau bzw. Rot dargestellt. Die Auswertung der Flächenanteile erfolgte nach Klassen $(0,05-0,1 \mathrm{~m} ; 0,1-0,5 \mathrm{~m}$ und $>0,5 \mathrm{~m}$ ), wobei Unterschiede $\pm 5 \mathrm{~cm}$ in den maximalen modellierten Wasserständen nicht explizit dargestellt wurden.

Grundsätzlich sind Abweichungen im Siedlungsgebiet größer als im Vorland. Dabei sind z. B. bei Nutzung ei- nes DGM ab $2,5 \mathrm{~m}$ bei mehr als $10 \%$ der Siedlungsfläche Abweichungen um mehr als $\pm 10 \mathrm{~cm}$ maximaler Einstauhöhe zu verzeichnen. Punktuell große Differenzen zwischen den Ergebnissen der Rechenläufe mit verschiedenen Auflösungen in den FloodArea-Simulationen ergeben sich teilweise zudem aufgrund der rasterbasierten Darstellung der Gebäudekanten.

Flexible Rechennetze, bei denen die Geometrien von Strukturen nahezu unabhängig von der gewählten Netzauflösung lagerichtig abgebildet werden können, führen nicht zu diesen Differenzen und haben diesbezüglich Vorteile. Ein limitierender Faktor dabei ist jedoch die Rechenzeit, welche meist höher ist als bei rasterbasierten Modellen. Zur Optimierung der Rechenzeiten besteht die Möglichkeit, unterschiedliche Auflösungen im Siedlungsbereich und im Vorlandbereich anzuwenden. Abb. 11 zeigt beispielhaft Ergebnisse einer derartigen Simulation bei der die Netzauflösung im Freilandbereich (oberhalb der Kontrollquerschnitte) von $0,5 \mathrm{~m}$ (links) auf $10 \mathrm{~m}$ (rechts) vergröbert wurde und die Auflösung im Siedlungsbereich (unterhalb der Kontrollquerschnitte) beibehalten wurde. Die Vergröberung des Netzes im Freilandbereich führt jedoch $\mathrm{zu}$ einer signifikanten Verlagerung des Abflusses an den beiden Kontrollquerschnitten im Gebiet (siehe Abb. 12).

\subsubsection{Variation der Rauheit}

Die in Abb. 13 und 14 dargestellten Ergebnisse basieren auf Simulationen mit Hydro_AS-2D im Gebiet Seewalchen. Erwartungsgemäß konnte beobachtet werden, dass bei rauer Parametrisierung die maximalen Überflutungshöhen tendenziell zunehmen und die Fließgeschwindigkeiten abnehmen. Lokal kann es aufgrund sich ändernder Fließwege dennoch $\mathrm{zu}$ davon abweichenden Ergebnissen kommen. Abb. 15 zeigt die Ergebnisse des gleichen Vergleichs zwischen dem rau parametrisierten Szenario und der Referenzvariante mit der Software FloodArea.

Die Ergebnisse aus den durchgeführten Simulationen in Schwertberg und Seewalchen mit Hydro_AS-2D und FloodArea zeigen auch auf, dass je nach modelltechnischer Umsetzung (Numerik, hydraulische Grundgleichungen) die Sensitivität der Modellergebnisse gegenüber verschiedenen Parametrisierungen des Fließwiderstands durchaus unterschiedlich sein kann. Betrachtet man die Abflüsse und Wasserstände aus Hydro_AS-2D, kommt es zu Erhöhungen der maximalen Wasserstände in den Hauptabflusswegen (Abb. 13). In den Ergebnissen aus FloodArea hingegeben ergibt sich ein differenziertes Bild (Abb. 15). Hintergrund dafür ist, dass ein Teil des Niederschlags bereits in den Hangflächen zurückgehalten wird und stark verzögert bzw. nicht innerhalb des Betrachtungszeitraums die Hauptabflusswege erreicht. Die Modelle reagieren unterschiedlich auf eine Variation der Fließwiderstandsparameter.

\subsubsection{Durchlässe}

Abb. 16 zeigt Differenzen in der maximalen Fließtiefe zwischen einem Referenzszenario (keine Durchlässe) und vier Varianten mit unterschiedlicher Durchlassdimensionierung, in Abb. 17 ist eine vergleichende Auswertung für acht Varianten dargestellt. Die hier dargestellten Ergebnisse wurden mit der Software Hydro_AS-2D berechnet und beziehen sich auf das Regenszenario mit $62 \mathrm{~mm} / \mathrm{h}$.

Aus den Abb. 16 und 17 ist ersichtlich, dass mit der verwendeten Methodik erst für Durchlässe ab DN 500 flächenhaft Wassertiefen um mehr als $5 \mathrm{~cm}$ reduziert werden. Bis DN 500 bewegen sich die Wassertiefendifferenzen allesamt im Bereich unter $\pm 5 \mathrm{~cm}$. Ab 


\section{Originalarbeit}
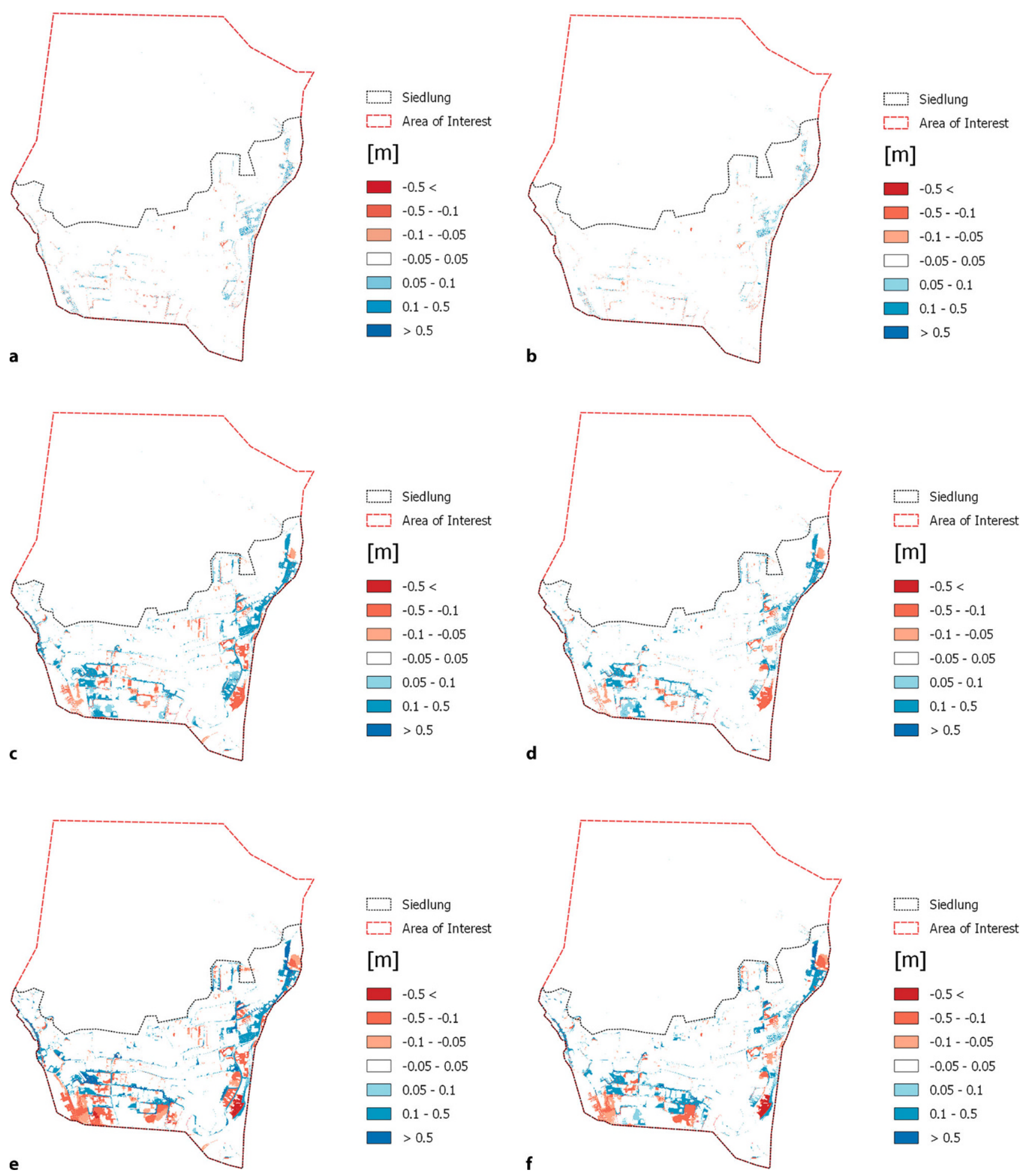

Abb. 18 Differenzen der maximalen modellierten Fließtiefen [m] für Modellvarianten mit drei Mauerhöhen $[10 \mathrm{~cm}, 50 \mathrm{~cm}, 100 \mathrm{~cm}]$, jeweils geschlossen (a, c, e) bzw. mit Öffnungen (b, d, f) im Vergleich zu einem Referenzszenario ohne Mauern. Software FloodArea, Regeninput $62 \mathrm{~mm} / \mathrm{h}$ für $1 \mathrm{~h}$, Simulationsdauer $2 \mathrm{~h}$. a Mauerhöhe $10 \mathrm{~cm}$ geschlossen, b Mauerhöhe $10 \mathrm{~cm}$ offen, $\mathbf{c}$ Mauerhöhe $50 \mathrm{~cm}$ geschlossen, d Mauerhöhe $50 \mathrm{~cm}$ offen, e Mauerhöhe $1 \mathrm{~m}$ geschlossen, $\mathbf{f}$ Mauerhöhe $1 \mathrm{~m}$ offen 


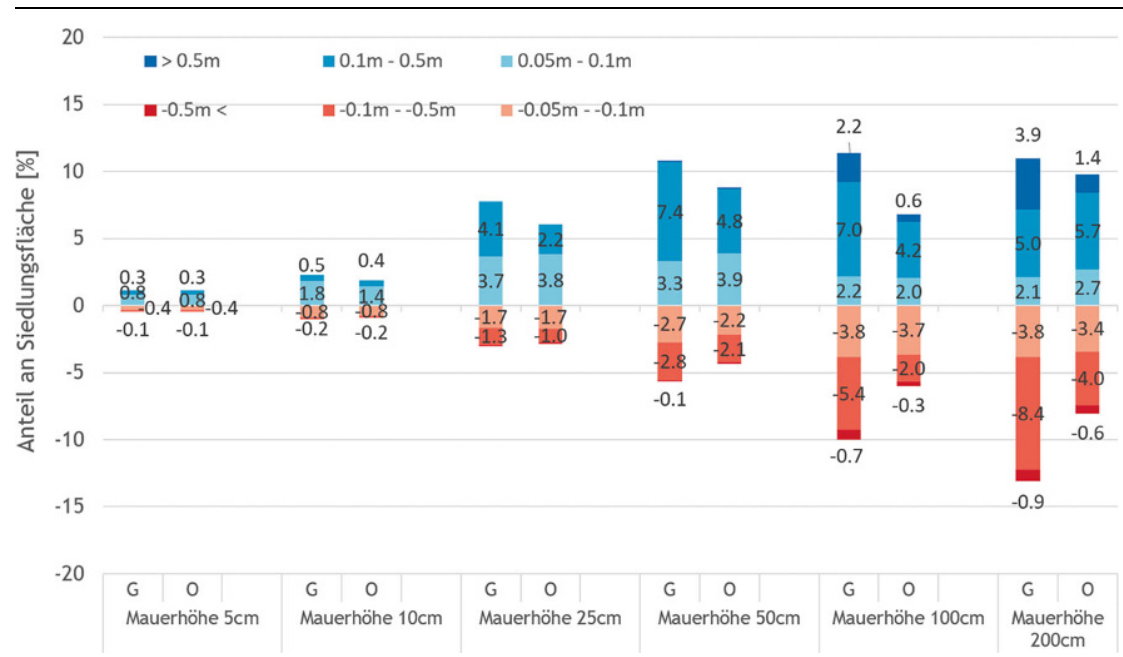

Abb. 19 Differenzen der maximalen modellierten Fließtiefen [m] für Modellvarianten mit sechs Mauerhöhen $[5 \mathrm{~cm}, 10 \mathrm{~cm}, 25 \mathrm{~cm}, 50 \mathrm{~cm}, 100 \mathrm{~cm}, 200 \mathrm{~cm}$ ], jeweils geschlossen $(G)$ bzw. mit Öffnungen $(O)$ im Vergleich zu einem Referenzszenario ohne Mauern. Software FloodArea, Regeninput $62 \mathrm{~mm} / \mathrm{h}$ für $1 \mathrm{~h}$, Simulationsdauer $2 \mathrm{~h}$

DN 500 sind für das betrachtete Beispiel Differenzen sowohl im Einlaufbereich der Durchlässe, als auch lokal in unterhalb des Einlaufs gelegenen Bereichen erkennbar. Ab einem Durchmesser von DN $600 \mathrm{~mm}$ sinken lokal die Wassertiefen um bis zu $10 \mathrm{~cm}$. Bei einem Durchmesser von DN 1000 werden bei einem der Durchlässe im Einlaufbereich mehr als $50 \mathrm{~cm}$ Wassertiefe reduziert. Drei der eingeführten Durchlässe zeigen überhaupt keine sichtbare Wirkung (d.h. max. Wasserstände ändern sich nur im Bereich unter $\pm 5 \mathrm{~cm}$ ) für das $62-\mathrm{mm} / \mathrm{h}$ Szenario.

\subsubsection{Fließhindernisse und Mauern}

Beispielhaft für die untersuchten Szenarien werden hier Ergebnisse der Berechnungen mit FloodArea vorgestellt. Abb. 18 zeigt Differenzraster für die Modellvarianten mit geschlossenen und offenen Mauern (Mauerhöhen 10, 25, $100 \mathrm{~cm}$ ) bei $62-\mathrm{mm} / \mathrm{h}$-Regenintensität im Vergleich zur Variante ohne Mauern. In Abb. 19 ist ein Vergleich für alle Mauerhöhen bei $62 \mathrm{~mm} / \mathrm{h}$ dargestellt. Es zeigt sich, dass bis zu einer Mauerhöhe von $10 \mathrm{~cm}$ die Differenzen flächig großteils im Bereich $\pm 5 \mathrm{~cm}$ bleiben, und nur in kleineren Bereichen größere Differenzen (bis $10 \mathrm{~cm}$ ) im Vergleich zur Variante ohne Mauern auftreten. $\mathrm{Ab}$ einer Mauerhöhe von $25 \mathrm{~cm}$ treten auch großflächig größere Abweichungen im Vergleich zum Referenzszenario auf, diese fallen mit steigender Mauerhöhe stärker aus. Wie zu erwarten, ergeben sich für die Szenarien mit Öffnungen kleinere Abweichungen zum Referenzszenario als bei durchgängigen Grundstückseinfriedungen.

Die Ergebnisse der Variantenstudien mit Hydro_AS-2D und JFlow (ausgewählte Szenarien) zeigen ein vergleichbares Bild: Mauerhöhen $\leq 10 \mathrm{~cm}$ verursachen - bezogen auf die modellierten Maximalwasserstände - in großen Teilen des Untersuchungsgebiets nur in wenigen Bereichen Differenzen von mehr als $\pm 5 \mathrm{~cm}$.

\section{Diskussion und Schlussfolgerung}

\subsection{Niederschlagsszenarien und Klimawandel}

Durch den Klimawandel ist nicht nur mit höheren Temperaturen, sondern auch mit höherer Intensität von Niederschlagsereignissen zu rechnen. Der mögliche Einfluss zukünftiger klimatischer Entwicklungen auf Überflutungen infolge von Starkniederschlagsereignissen wurde in der RAINMANPilotstudie Oberösterreich nicht dezidiert untersucht, entsprechende Untersuchungen für ausgewählte Gebiete in Oberösterreich wurden jedoch in den beiden Projekten SAFFER-CC und AQUACLEW (https://aquaclew.eu) durchgeführt (Strehz et al. 2015; Lumassegger et al. 2016a; Achleitner et al. 2019).

Je extremer dabei das Niederschlagsereignis (d.h. je geringer dessen Auftretenswahrscheinlichkeit bzw. grö- ßer dessen Wiederkehrperiode) und je kürzer die Niederschlagsdauer, desto stärker könnten die Intensitäten der Niederschlagsereignisse im Vergleich zur aktuellen Situation steigen. Niederschläge mit Dauerstufen von $60 \mathrm{~min}$ oder weniger könnten dabei pro Grad Celsius an Temperatursteigerung um bis $\mathrm{zu} 10 \%$ intensiver ausfallen als unter aktuellen Bedingungen. Für Niederschlagsereignisse mit längerer Dauer liegen mögliche Steigerungsraten aus derzeitiger Sicht um die $7 \% /{ }^{\circ}$ Celsius oder darunter. Diese Einschätzungen decken sich überwiegend mit Ergebnissen und Einschätzungen aus verschiedenen Publikationen zum Thema (u. a. Lenderik und van Meijgaard 2008; Formayer und Kromp-Kolb 2009; Berg et al. 2013; Formayer und Fritz 2017; Giorgi et al. 2019; Becker 2019). Basierend auf diesen Annahmen ist davon auszugehen, dass in Zukunft die Anzahl betroffener Gebäude und überfluteter Flächen bei ähnlichen Vorbedingungen in den betroffenen Gebieten noch deutlich größer sein könnte als unter aktuellen Randbedingungen.

Eine explizite Berücksichtigung möglicher Klimawandelauswirkungen bei der Hangwassermodellierung für $\mathrm{Be}$ messungsfragen und Gefahrenhinweiskarten (Hangwasserhinweiskarten) erfolgt aktuell nicht. Die formalen und rechtlichen Grundlagen dafür sind noch nicht in ausreichendem Maße gegeben, um Klimawandelaspekte bei Starkniederschlägen $\mathrm{zu}$ berücksichtigen. In der RIWA-T (2016), Abschn. 4.8 wird festgehalten, dass im Zuge der Risiko- und Restrisikoabschätzung die „möglichen Auswirkungen des Klimawandels auf die Hochwassergefahren durch geeignete wasserwirtschaftliche Untersuchungen in regelmäßigen Zeitabständen abzuschätzen" sind. Klimaänderungen auch in die Gefährdungsabschätzung von pluvialen Hochwassersituationen einzubeziehen, wird zunehmend wichtig (Wiedner et al. 2020). Aktuell wurde dem durch eine entsprechende Aktualisierung der Bemessungsniederschläge Rechnung getragen (Weilguni 2019). Abb. 20 verdeutlich die in vielen Gebieten verzeichnete Zunahme der 100-jährlichen 60-Minuten-Bemessungsniederschläge $\left(\mathrm{N}_{100,60}\right)$.

Die in diesem Beitrag vorgestellten Berechnungsergebnisse zeigen vor allem die unterschiedlichen Auswirkungen hydraulisch relevanter Strukturen wie Durchlässe und Mauern auf das 
Differenz BemessungsNS 2020-2009

$\mathrm{d}=60 \mathrm{~min}, \mathrm{t}=100 \mathrm{j}$.

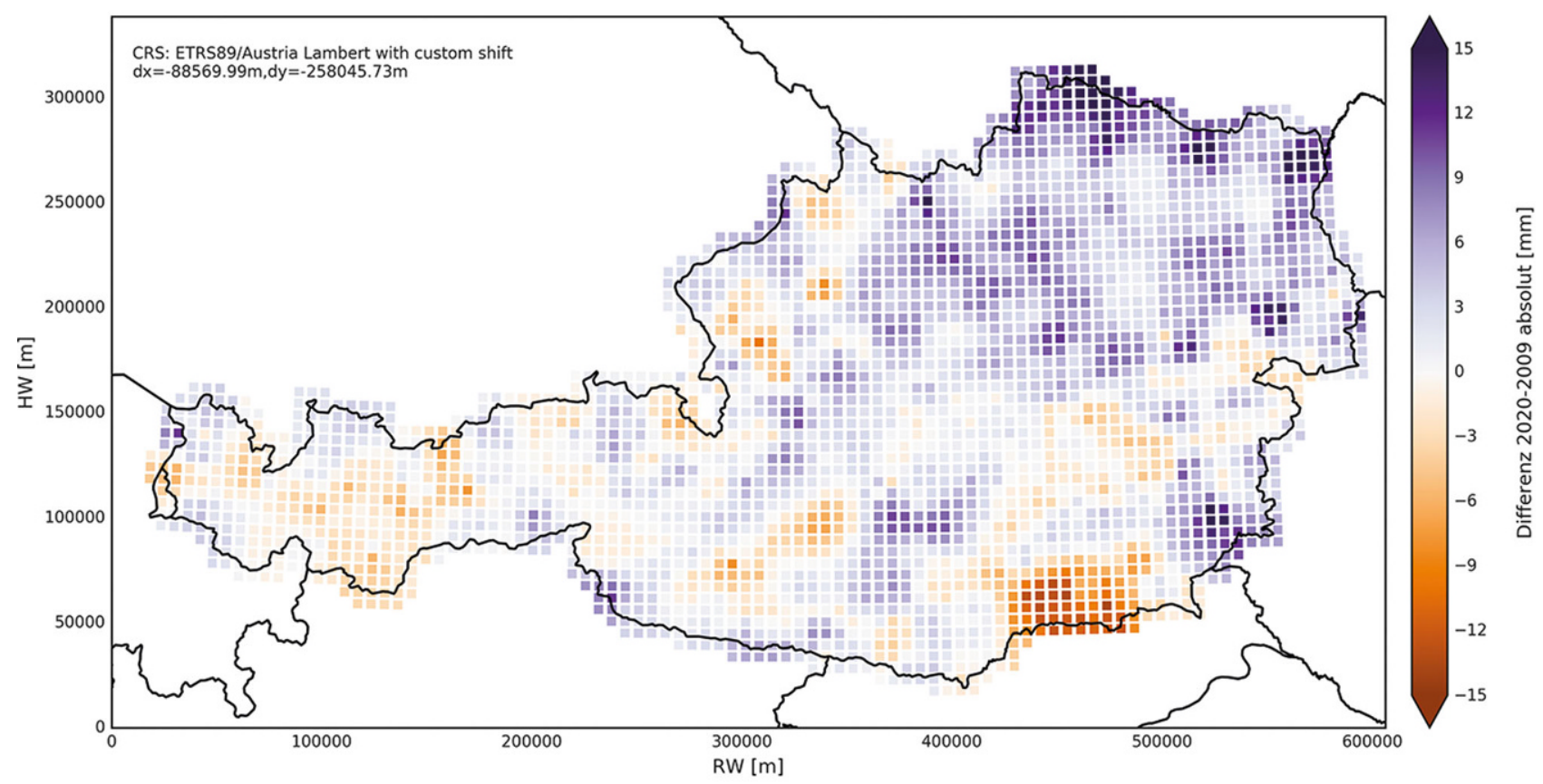

Differenz BemessungsNS 2020-2009 $\mathrm{d}=60 \mathrm{~min}, \mathrm{t}=100 \mathrm{j}$.

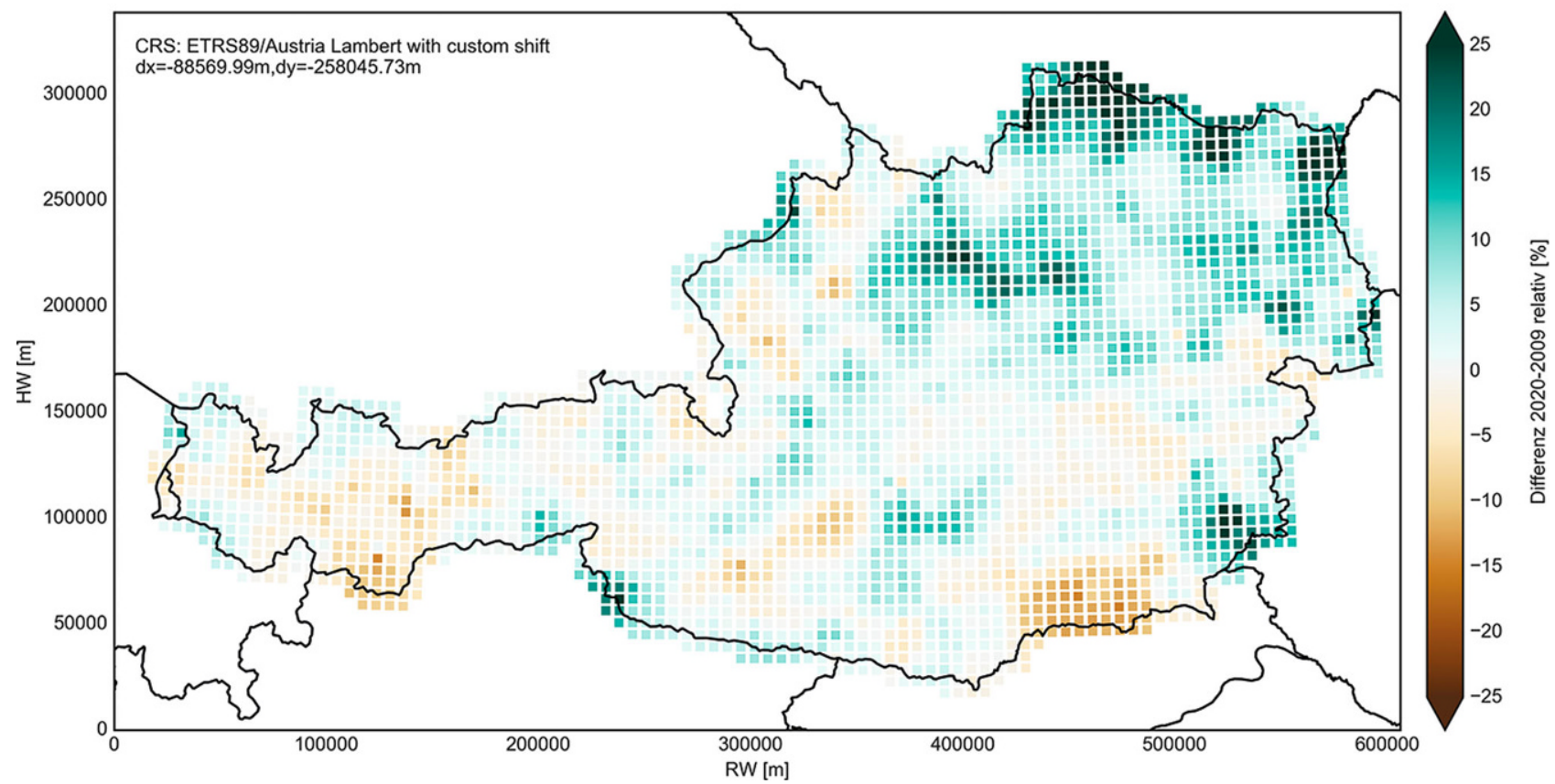

Abb. 20 Absolute (oben, mm) und relative (unten, \%) Unterschiede der Bemessungsniederschlagswerte (NBemess) für einen Niederschlag mit $1 \mathrm{~h}$ Dauer und 100-jährlichem Wiederkehrintervall zwischen 2009 und 2020 (Daten von ehyd.gv.at)

Abflussgeschehen bei verschiedenen Ereignissen, was - zusammen mit der Klimawandel-Diskussion in diesem Kapitel - eine Prüfung verschiedener Szenarien zur Gefährdungsabschätzung und Maßnahmenwirksamkeit sinnvoll 5.2 Abflussbildung erscheinen lässt.

Die Abflussbildung auf unterschiedlichen Flächen ist u.a. abhängig von Faktoren wie der aktuellen Vegetationsbedeckung, dem Versiegelungsgrad, 
der Landnutzung und den Bodeneigenschaften. Die meisten dieser Faktoren sind saisonalen und auch langfristigen Änderungen unterworfen. In Abhängigkeit von den aktuell vorherrschenden Verhältnissen kann es bei vergleichbaren Niederschlägen zu unterschiedlich ausgeprägter Oberflächenabflussbildung kommen.

Ein pragmatischer Ansatz, mit dieser Variabilität umzugehen, ist die Verwendung entsprechender regionalmaßstäblicher Abflussbeiwertkarten oder Nettoregen-Datensätze (vgl. LUBW 2016; Steinbrich et al. 2016; Sotier et al. 2017). Die dahinterstehenden Annahmen zur Abflussbereitschaft von Teilflächen (Vorbedingung, Boden/ Landnutzung) stellt eine einheitliche Festlegung dar, um vergleichbare Modellrechnungen in unterschiedlichen Gebieten zu gewährleisten.

Forschungsbedarf ergibt sich in diesem Zusammenhang hinsichtlich der kombinierten Auftretenswahrscheinlichkeit von extremen Niederschlagsereignissen und den (kurzfristigen bzw. saisonalen) Vorbedingungen.

\subsection{Abflusskonzentration-2D-HN- Modellierung}

\subsubsection{Modellauflösung}

Für die Modellierung von Oberflächenabfluss ist eine detaillierte Abbildung der Geländeoberfläche und hydraulisch relevanter künstlicher und natürlicher Strukturen besonders wichtig. Sie dient dazu, das Abflussverhalten an der Oberfläche möglichst realitätsnah abzubilden. Für die Modellierung wird daher ein DGM mit einer Auflösung von $\leq 1 \mathrm{~m}$ empfohlen. Ab einer Rasterauflösung gröber $1 \mathrm{~m}$ können z.B. die Geometrien von Gebäuden oder anderen hydraulisch relevanten Strukturen nur mehr unzureichend genau abgebildet werden.

Bei flexiblen Netzen sollte unabhängig von der gewählten Netzauflösung für die Abbildung der Höhen immer ein möglichst hochaufgelöstes DGM genutzt werden. Bei Vergrößerung der Netzelemente außerhalb des Siedlungsbereiches ist darauf $\mathrm{zu}$ achten, dass die durch die Geländeform vorgegebenen Fließwege, wie z.B. Wege oder Straßen in Hanglage, aber auch kleine Gräben erhalten bleiben, die die Fließrichtung maßgeblich verändern können. Die Untersuchungen legen nahe, dass eine Netzauflösung außerhalb des
Siedlungsbereiches gröber als 2,5 $\mathrm{m}$ die Fließwege maßgeblich verändern und somit grundsätzlich nicht empfohlen werden kann.

\subsubsection{Hydraulisch relevante Strukturen}

Im Ereignisfall können im Siedlungsbereich Grundstückseinfriedungen in Form von Zäunen, Mauern oder Hecken die lokalen Strömungsverhältnisse beeinflussen. Mauern bis zu einer Höhe von $10 \mathrm{~cm}$ zeigten keine relevanten Auswirkungen auf die maximalen modellierten Wassertiefen in den untersuchten Siedlungsgebieten. Das Amt der Oberösterreichischen Landesregierung empfiehlt, bei der Modellierung von Oberflächenabfluss für Starkregenereignisse Mauern bis $10 \mathrm{~cm}$ und damit auch Bordsteine näherungsweise zu vernachlässigen. Werden Mauern im Modell eingebaut, sollten aufgrund der nachgewiesenen Wirkung auf das Abflussgeschehen vorhandene Öffnungen (Einfahrten, Gartentore) möglichst realitätsnah berücksichtigt werden.

Inwieweit es sinnvoll ist, Durchlässe wie z.B. Verrohrungen oder Unterführungen in der hydrodynamischen Modellierung zu berücksichtigen, hängt vom betrachteten Szenario und der Dimension des Durchlasses ab. In der Praxis muss damit gerechnet werden, dass Durchlasseinläufe je nach Durchmesser, Lage und baulicher Ausführung im Ereignisfall nur eine beschränkte Wirksamkeit aufweisen können (Achleitner et al. 2020b, c). Durchlässe bis $\mathrm{zu}$ einem Durchmesser DN 300 haben selbst bei voller Wirksamkeit nur geringfügige Auswirkungen auf die Simulationsergebnisse.

Das Amt der Oberösterreichischen Landesregierung empfiehlt, Durchlässe größer DN 300 im Modell zu berücksichtigen und bei Implementierung von Durchlässen auf lokaler Detailebene die hydraulische Wirksamkeit bei Extremereignissen vor Ort zu überprüfen. Bei Berücksichtigung von Durchlässen im Modell sollten auch Überflutungsszenarien infolge Verklausung von Einlaufbauwerken betrachtet werden.

\subsubsection{Rauheiten}

Ergebnisse aus den untersuchten Pilotgebieten Schwertberg und Seewalchen zeigen auf, dass je nach modelltechnischer Umsetzung (Numerik, hydraulische Grundgleichungen) die Sensitivität der Modellergebnisse ge- genüber verschiedenen Parametrisierungen des Fließwiderstandes durchaus unterschiedlich sein kann. Eine rauere Parametrisierung für die betrachteten Beispiele führte dabei erwartungsgemäß jeweils $\mathrm{zu}$ tendenziell höheren simulierten max. Wassertiefen und geringeren max. Fließgeschwindigkeiten; eine im Vergleich dazu glattere Parametrisierung hat den gegenteiligen Effekt.

$\mathrm{Zu}$ erwähnen ist, dass eine konstante Rauheit immer eine Vereinfachung darstellt. Neben der saisonalen Änderung der Fließwiderstände besteht eine $\mathrm{Ab}$ hängigkeit des Fließwiederstandes von der Wassertiefe.

Die hier dargestellten Ergebnisse legen nahe, dass für Gefährdungsabschätzungen und Maßnahmenplanungen verschiedene Szenario-Modellierungen sinnvoll sein können, um die Wirksamkeit der Maßnahme auch unter veränderten Bedingungen bestmöglich sicherstellen zu können.

Danksagung Die vorgestellte Studie beruht auf Arbeitsinhalten der Projekte RAINMAN, AQUACLEW und SAFFER.

Die Studie „RAINMAN, PILOTSTUDIE OBERÖSTERREICH, MODELLIERUNG VON STARKREGEN-OBERFLÄCHENABFLUSS/HANGWASSER“ (Achleitner et al. 2020b, c) wurde durch das Umweltbundesamt als Projektpartner beauftragt und zusätzlich vom Amt der Oberösterreichischen Landesregierung als assoziiertem Partner inhaltlich-fachlich begleitet. Das Projekt RAINMAN CE 986 wurde mit Mitteln aus dem Interreg Central Europe-Programm der Europäischen Union und dem Bundesministerium für Landwirtschaft, Regionen und Tourismus finanziert.

Das Projekt AQUACLEW wird durch die European Research Area for Climate Services (ERA4 CS) im Rahmen der Joint Programming Initiative „Connecting Climate Knowledge for Europe“ (JPI Climate) finanziert. Dabei handelt es sich um eine Zusammenarbeit zwischen der Europäischen Kommission und den nationalen Forschungsräten der Mitgliedstaaten, die sich die Kosten teilen (AQUACLEW Grant Nr. 690462).

Das Projekt SAFFER-CC „Sensitivity Assessment of critical condition for local Flash Floods - Evaluating the Recurrence under Climate Change (Nr. KR14AC7K11882)“ wurde im Rahmen des Austrian Climate Research Program 
(ACRP) des Klima- und Energiefonds gefördert (Achleitner et al. 2019).

Im Rahmen dieser Studien wurden Berechnungen mit verschiedenen hydrodynamischen Softwarepaketen durchgeführt. Der Universität Innsbruck wurden dankenswerterweise Lizenzen für Hydro_AS-2D von der Firma Hydrotec und Lizenzen für FloodArea von der Firma Geomer für die Berechnungen zur Verfügung gestellt. Weiters wurden für ausgewählte Szenarien Berechnungen mit der Software JFLOW durch die Brandverhütungsstelle Oberösterreich durchgeführt.

Funding Open access funding provided by University of Innsbruck and Medical University of Innsbruck.
Open Access Dieser Artikel wird unter der Creative Commons Namensnennung 4.0 International Lizenz veröffentlicht, welche die Nutzung, Vervielfältigung, Bearbeitung, Verbreitung und Wiedergabe in jeglichem Medium und Format erlaubt, sofern Sie den/die ursprünglichen Autor(en) und die Quelle ordnungsgemäß nennen, einen Link zur Creative Commons Lizenz beifügen und angeben, ob Änderungen vorgenommen wurden.

Die in diesem Artikel enthaltenen Bilder und sonstiges Drittmaterial unterliegen ebenfalls der genannten Creative Commons Lizenz, sofern sich aus der Abbildungslegende nichts anderes ergibt. Sofern das betreffende Material nicht unter der genannten Creative
Commons Lizenz steht und die betreffende Handlung nicht nach gesetzlichen Vorschriften erlaubt ist, ist für die oben aufgeführten Weiterverwendungen des Materials die Einwilligung des jeweiligen Rechteinhabers einzuholen.

Weitere Details zur Lizenz entnehmen Sie bitte der Lizenzinformation auf http://creativecommons.org/licenses/ by/4.0/deed.de.

\section{Literatur}

Achleitner, S., Kohl, B., Lumassegger, S., Huber, A., Formayer, H. und Weingraber, F. (2020a): Sturzfluten, In Thomas Glade, Martin Mergili, Katrin Sattler (Hrsg.) ExtremA 2019. Aktuelle Wissensstand zu Extremereignissen alpiner Naturgefahren in Österreich. Vienna University Press, S. 247-286, ISBN Print: $9783847110927-$ ISBN E-Lib: 9783737010924

Achleitner, S., Huber, A., Lumassegger, S., Kohl., B., Spira, Y., Weingraber, F. (2020b): Pilot study Upper Austria-Modeling of pluvial floods in response to heavy precipitation events-Guidance for Practitioners. URL: https://www.interregcentral.eu/Content.Node/Home/Projects/RAIN MAN/Main-Project-outputs-/RAINMAN-LeitfadenEN-V1.0-20200623-(1).pdf. (Zugriff: 19.02. 2021).

Achleitner, S., Huber, A., Lumassegger, S., Kohl., B., Spira, Y., Weingraber, F. (2020c): Pilotstudie Oberösterreich - Modellierung von StarkregenOberflächenabfluss/Hangwasser. Techn. Bericht. Projekt RAINMAN. URL: https://www. interreg-central.eu/Content.Node/Home/Proje cts/RAINMAN/Main-Project-outputs-/AU-TechnischerBericht.pdf. (Zugriff: 19.02.2021).

Achleitner, S., Lumassegger, S., Kohl, B., Formayer, H., Leidinger, D., Strehz, A., Einfalt, T. (2019): SAFFER-CC, Sensitivity assessment of critical condition for local flash floods, Austrian Climate Research Programme-7th Call (B464786);Publizierbarer Endbericht; https:// www.klimafonds.gv.at/wp-content/uploads/ sites/6/B464786-ACRP7-SAFFER-CC-KR14AC7 K11882-EB pdf. (Zugriff: 19.02.2021.)

Becker, A. (2019): Extremerer Niederschlag im Klimawandel - Was wissen wir? WasserWirtschaft, 12:10-16.

Berg, P., Moseley, C., Haerter, J. (2013): Strong increase in convective precipitation in response to high temperatures. Nature Geoscience, 6:181185. URL https://doi.org/10.1038/ngeo1731.

Bernet, D. B., Prasuhn, V. \& Weingartner, R (2017): Surface water floods in Switzerland what insurance claim records tell us about the damage in space and time Natural Hazards and Earth System Sciences, 17, 1659-1682

Bernet, D. B., Zischg, A., Prasuhn, V., Weingartner, R. (2018): Modeling the extent of surface water floods in rural areas: Lessons learned from the application of various uncalibrated models. Environmental Modelling \& Software, 109:134-151

Broich, K., Kaiser, M., Lin, Q., Mitterer, J., Nguyen, H., Pflugbeil, T., von Trentini, F., Willkofer, F., Disse, M., Ludwig, R. (2018): Das Projekt HiOS - Erstellung einer Hinweiskarte für Oberflächenabfluss und Sturzfluten für bayerische Gemeinden. In: Disse, M., Kaiser, M. (Hg.) Starkregen und Sturzfluten - Erfassen, Erforschen, Evaluieren; Beiträge zum Seminar am 6. Juni 2018, Nummer 40.18 in Forum für $\mathrm{Hy}^{-}$ drologie und Wasserbewirtschaftung, S. 27-40 https://doi.org/10.14617/for.hydrol.wasbew.40.

Chow, V. T. (1959): Open-channel hydraulics. McGraw-Hill, New York.

Courty, L. G., Pedrozo-Acuña, A., Bates, P. D. (2017): Itzï (version 17.1): an open-source, distributed GIS model for dynamic flood simulation. Geoscientific Model Development, 10:1835-1847.

Engman, E. T. (1986): Roughness coefficient for routing surface runoff. Journal of Irrigation and Drainage Engineering 112:39-53.

Formayer, H. and A. Fritz (2017): „Temperature dependency of hourly precipitation intensities-surface versus cloud layer temperature." International Journal of Climatology 37(1): 1-10. Formayer, H. \& Kromp-Kolb, H. (2009): Hochwasser und Klimawandel. Auswrikungen des Klimawandels auf Hochwasserereignisse in Österreich Institut für Meteorologie (BOKU-Met), Department für Wasser - Atmosphäre - Umwelt, Universität für Bodenkultur Wien, 2009

Giorgi, F., Raffaele, F., Coppola, E. (2019): The response of precipitation characteristics toglobal warming from climate projections. Earth System Dynamics, 10:73-89. URL https://doi org/10.5194/esd-10-73-2019.

Holmgren, P. (1994): Multiple flow direction algorithms for runoff modelling in grid based elevation models: an empirical evaluation Hydrological Processes, 8, 327-334

Humer, G., Reithofer, A., Klar, R., Achleitner, S. (2015): Erweiterung eines 2D-Strömungsmodelles zur Berechnung von Sturzfluten. Plausibilisierung und praktische Anwendung für die Darstellung von Gefahrenkarten und Lösung von Planungsaufgaben in Siedlungsgebieten. Korrespondenz Wasserwirtschaft, 8(3), S. 164-171.
Hydrotec (2019): Hydro AS-2D - 2D- Strömungsmodell für die wasserwirtschaftliche Praxis. Hydro_AS-2D Benutzerhandbuch Version 5.1.0

Kipfer, A., Kienholz, C., Liener, S. (2012): Ein neuer Ansatz zur Modellierung von Oberflächenabfluss. In: 12th Congress INTERPRAEVENT 2012, Grenoble/France, Conference Proceedings, S. 179-189.

Kipfer, A., Berger, C., Mani, P., Hulliger, R., Caduff, U., Dobmann, J., Gsteiger, P., Pauli, M. \& Schneider, M. (2018): Gefährdungskarte Oberflächenabfluss Schweiz. Technischer Bericht, geo7, Bern, Schweiz. https://www.bafu.admin $\mathrm{ch} / \mathrm{dam} / \mathrm{bafu} / \mathrm{de} /$ dokumente/naturgefahren/ externe-studienberichte/gefaehrdungskarteoberflaechenabfluss-schweiz-technischerbericht. pdf.download.pdf/gefaehrdungskarte_oberflae chenabfluss_schweiz_technischer_bericht.pdf. (Zugriff: 19.02.2021)

Klar, R., Achleitner, S., Lumassegger, S., Aufleger, M. \& Hofer, M. (2014): Extension and testing of a 2D hydrodynamic model for direct rainfall runoff-simulation, 11th International Conference on Hydroinformatics-HIC 2014

Kohl, B. (2011): Das Niederschlags-Abflussmodell ZEMOKOST. Entwicklung eines praktikablen Modells zur Ermittlung von Hochwasserabflüssen in Wildbacheinzugsgebieten unter Einbeziehung verbesserter Felddaten. Phd Thesis, Universität Innsbruck.

Lenderink, G., van Meijgaard, E. (2008): Increase in hourly precipitation extremes beyond expectations from temperature changes. Nature Geoscience, 1(8), S. 511-514.

LUBW (2016): Leitfaden Kommunales Starkregenrisikomanagement in Baden-Württemberg. Leitfaden, LUBW Landesanstalt für Umwelt, Messungen und Naturschutz Baden-Württemberg.

LUBW (2020): Anhänge 1 a,b,c zum Leitfaden Kommunales Starkregenrisikomanagement in Baden-Württemberg. Stand März 2020. LUBW Landesanstalt für Umwelt, Messungen und Naturschutz Baden-Württemberg (www.lubw. baden-wuerttemberg.de/wasser/starkregen) Lumassegger, S., Achleitner, S., Klar, R., Kohl, B. (2016a): Flash flood formation in pre-alpine landscape-Impact of the spatial distribution of storm event. In: Koboltschnig, Gernot (Ed.): 13th Congress IN-TERPRAEVENT 2016, 30 May to 2 
June 2016, Lucerne, Switzerland-Extended Abstracts. Klagenfurt: Internationale Forschungsgesellschaft Interpraevent / International Research Society Interpraevent, S. 128-29.

Lumassegger, S., Achleitner, S., Kohl, B., Formayer, H., Strehz, A., Einfalt, T. (2016b): Auswirkungen räumlich variabler Niederschläge auf die Sturzflutsimulation. In: Casper, Markus Gronz, Oliver (Hg.): Räumliche Heterogenität Erkennen, Abbilden, Validieren oder Ignorieren? Beiträge zum 4. Trierer Workshop zur Niederschlag-Abfluss-Modellierung am 5./6. Oktober 2015in Trier. Hennef: FgHW - Forum für Hydrologie und Wasserbewirtschaftung, Heft 36.16 ISBN 978-3-88721-341-1, S. 131-139. https:// doi.org/10.14617/for.hydrol.wasbew.36

Maniak, U. (2010): Hydrologie und Wasserwirtschaft - Eine Einführung für Ingenieure. Springer. https://doi.org/10.1007/978-3-642-053962 .

Markart, G., Kohl, B., Sotier, B., Schauer, T., Bunza, G., \& Stern, R. (2005): Provisorische Geländeanleitung zur Abschätzung des Oberflächenabflussbeiwertes auf alpinen BodenVegetationseinheiten bei konvektiven Starkregen (Version 1.0)(A simple code of practice for assessment of surface runoff coefficients for alpine soil-/vegetation units in torrential rain (Version 1.0)). Bundesforschungszentrum für Wald, BFW-Dokumentation, 3.Jg

McCuen, R. H. (2016): Hydrologic Analysis and Design. Pearson Higher Education, Hoboken, USA, 4. Auflage.

Neelz, S.- und Pender, G. (2013): Benchmarking the latest generation of $2 \mathrm{D}$ hydraulic modelling packages, Report-SC120002. Environment Agency, Bristol, ISBN: 978-1-84911-306-9

Nissen, K. M. und Ulbrich, U. (2017): Increasing frequencies and changing characteristics of heavy precipitation events threatening in frastructure in Europe under climate change. Natural Hazards and Earth System Sciences, (17):1177-1190. https://doi.org/10.5194/nhess17-1177-2017

O'Callaghan, J. F. \& Mark, D. (1984): The Extraction of Drainage Networks from Digital Elevation
Data Computer Vision, Graphics and Image Processing, 28, 323-344

Quinn, P., Beven, K., Chevallier, P. \& Planchon, 0. (1991): The prediction of hillslope flow path for distributed hydrological modelling using digital terrain models Hydrological Processes, 5, 59-79

RIWA-T (2016): Technische Richtlinien für die Bundeswasserbauverwaltung RIWA-T, gemäß $\$ 3$ Abs. 2 WBFG Fassung 2016 GZ: UIW.3.3.3/0028IV/6/2015

Schubert, J. E., Sanders, B. F., Smith, M. J., Wright, N. G. (2008): Unstructured mesh generation and landcover-based resistance for hydrodynamic modeling of urban flooding. Advances in Water Resources, 31:1603-1621.

SCS (1972): National Engineering Handbook, Section 4; Soil Conservation Service USDA: Washington, DC, USA, 1972.

Sotier, B., Klebinder, K., Bauer, T., Markart, G. und Strauss, P. (2017): Hydrologische Bodenkenndaten Niederösterreich - HYDROBOD NÖ II, zweite Projektphase; Endbericht inkl. Ergänzungen; http://opendata.noe.gv.at/ogd-data/ BD3/hydrobod/Hydrobod2 final Bericht inkl Erg\%C3\%A4nzungen.odt. (Zugriff:19.02.2021). Starl, H. (2020): Hangwassermodellierungen und deren Möglichkeit zur Abschätzung von potenziellen Gefährdungen für Gebäude - Eine Analyse anhand von Starkregenereignissen in Oberösterreich. Bautechnik. https://doi.org/10. 1002/bate.201900028

Steinbrich, A., Leistert, H. \& Weiler (2016): M. Model-based quantification of runoff generation processes at high spatial and temporal resolution Environmental Earth Sciences, 75, 1423 Strehz, A., Einfalt, T., Achleitner, S., Lumassegger, S. (2015): Analysis of small scale convective precipitation events in Austria. In: 10th International Workshop on Precipitation in Urban Areas, Rainfall in Urban and Natural Systems. Zürich: Eidgenössische Technische Hochschule (ETH)

Tarboton, D. G. (1997): A new method for the determination of flow directions and upslope areas in grid digital elevation models Water Resources Research, 33, 309-319
Tyrna, B., Assmann, A., Fritsch, K., Johann, G. (2017): Large-scale high-resolution pluvial flood hazard mapping using the raster-based hydrodynamic two-dimensional model FloodAreaHPC. Journal of Flood Risk Management. https://doi. org/10.1111/jfr3.12287.

US Army Corps of Engineers-Hydrologic Engineering Center (2018): Benchmarking of the HEC-RAS Two-Dimensional Hydraulic Modeling Capabilities. Technical Report (https:// www.hec.usace.army.mil/ software/hec-ras/ documentation/RD-51_Benchmarking_2D.pdf) Weilguni, V. (2009): Bemessungsniederschläge in Österreich. Wiener Mitteilungen Band 216.

Weilguni, V. (2019): Vom Messwert zum Bemessungsniederschlag - ein Service der Hydrographie Österreichs. Österreichische Wasser- und Abfallwirtschaft, 71, 36-42.

Wiedner, J., Paal, W., Gobiet, A., Leidinger, D., Pistotnik, G., Strommer, G., Achleitner, S. Blöschl, G., Günther, M., Mehlhorn, S., Muschalla, D., Unterwaining, M., Hornich, R., Ankowitsch, A., Huber, H., Neuhold, C., Seher, W., Rauchlaitner, P., Falkensteiner, P., Haider, S., Haslinger, H., Starl, H. (2020): ÖWAVExpertInnenpapier „Klimawandelanpassung Wasserwirtschaft - Pluviales Hochwasser/Oberflächenabfluss“. Erstellt vom ÖWAV-Forum „Klimawandel“, Österreichischer Wasser- und Abfallwirtschaftsverband (ÖWAV), Wien, 2020

Zahnt, N., Eder, M. \& Habersack, H. (2018): Herausforderungen durch pluviale Überflutungen Grundlagen, Schäden und Lösungsansätze Österreichische Wasser- und Abfallwirtschaft, 70, $64-77$

Hinweis des Verlags Der Verlag bleibt in Hinblick auf geografische Zuordnungen und Gebietsbezeichnungen in veröffentlichten Karten und Institutsadressen neutral. 